\title{
A Mega-High-Throughput Screening Platform for the Discovery of Biologically Relevant Sequence-Defined Non-Natural Polymers
}

Michal Avital-Shmilovici, Xiaohe Liu, Thomas Shaler, Andrew Lowenthal, Pauline Bourbon, Janey Snider, Arlyn Tambo-Ong, Claire Repellin, Kenya Yniguez, Lidia Sambucetti, Peter B. Madrid ${ }^{*}$ and Nathan Collins*

SRI Biosciences Division, SRI International, Menlo Park, CA 94025 USA

*Email: peter.madrid@sri.com; nathan.collins@sri.com

\begin{abstract}
Combinatorial methods enable the synthesis of chemical libraries on scales of millions to billions of compounds, but the ability to efficiently screen and sequence such large libraries has remained a major bottleneck for molecular discovery. We developed a novel technology for screening and sequencing libraries of synthetic molecules of up to a billion compounds in size. This platform utilizes the Fiber-optic Array Scanning Technology (FAST) to screen bead-based libraries of synthetic compounds at a rate of 5 million compounds per minute $(\sim 83,000 \mathrm{~Hz})$. This ultra-highthroughput screening platform has been used to screen libraries of synthetic "self-readable" nonnatural polymers that can be sequenced at femtomole scale by chemical fragmentation and highresolution mass spectrometry. The versatility and throughput of the platform was demonstrated by screening two libraries of non-natural polyamide polymers with sizes of $1.77 \mathrm{M}$ and $1 \mathrm{~B}$ compounds against the protein targets K-Ras, asialoglycoprotein receptor 1 (ASGPR), IL-6, IL-6
\end{abstract}


receptor (IL-6R) and TNFa. Hits with low nanomolar binding affinities were found against all targets, including competitive inhibitors of K-Ras binding to Raf and functionally active uptake ligands for ASGPR facilitating intracellular delivery of a non-glycan ligand.

\section{Introduction}

Natural biological polymers such as peptides, proteins, and nucleic acids have evolved molecular recognition functionalities to produce highly specific binding interactions and catalytic enzymatic functions. In an empirical approach to discover novel affinity agents, catalysts, and materials, biotechnologists have exploited these rich functional properties to create therapeutics, diagnostics, sensors, industrial reagents, and biomedical research probes by using powerful biological tools to screen large libraries of natural polymers of $10^{7-12}$ unique molecular sequences. ${ }^{1}$ As data regarding the structures of biopolymers have amassed, so has the ability to rationally design de novo proteins and other biopolymers. ${ }^{2}$

There is a growing interest in expanding polymer discovery into the field of sequence-defined non-natural polymers or foldamers ${ }^{3}$ using chemical rather than biological synthesis to further access molecular diversity in novel 3D folded structures with related functionality as affinity reagents, therapeutics and catalysts. ${ }^{4}$ At present, there is insufficient sequence and related structural data to design non-natural polymers de novo; as such, screening libraries to find polymers with desired properties is currently the most practical solution.

Chemical synthetic methodology for building libraries of peptide-based polymers is wellestablished on solid support beads via the "one-bead one-compound" (OBOC) method. ${ }^{5}$ This combinatorial "mix and split" method has the potential to make chemical polymer libraries of similar size and complexity to biological methods; however, in practice, it has traditionally been 
used to produce relatively small libraries on the scale of only thousands to hundreds of thousands of compounds because of the two major hurdles of library screening and hit sequencing. ${ }^{6}$ OBOC libraries can be synthesized with total chemical control and the relatively harsh chemical reaction conditions of organic synthesis are well tolerated compared to more biological library production methods. ${ }^{7}$ To expand the application of OBOC libraries to the discovery of sequence defined nonnatural polymers it is necessary to address the constraints of $\mathrm{OBOC}$ screening throughput for appropriately scaled molecular diversity and the requirement to handle low (ideally picomole or lower) amounts of hit compounds for sequence identification.

The current commercially available technology with the highest throughput for bead screening is fluorescence-activated cell sorting (FACS $)^{8}$, which has a theoretical throughput of $\sim 10^{8}$ in a 10 hour period. In practice, however, hit enrichment from large libraries $\left(>10^{6}\right)$ using pull-down methods is the only way to achieve time- and cost-effective FACS screening that reduces the number of beads to $10^{4-5} .8 \mathrm{~b}$ An alternative approach described by Carney et al. ${ }^{9}$ used confocal laser scanning microscopy (CLSM) to screen beads immobilized on a $10 \mathrm{~cm} \times 10 \mathrm{~cm}$ polystyrene surface. Autofluoresence of each bead $\left(F_{0}\right)$ was measured first and compared with the fluorescence of labeled beads during screening $(\Delta F)$ to identify the brightest beads $\left(\Delta F / F_{0}\right)$. By this approach they could screen 200,000 beads in 20 mins in this fluorometric assay and demonstrated its application in a multiplexed screen of 157,423 beads from a 9-mer peptide library on $90 \mu \mathrm{m}$ beads. Setting the hit rate at $0.01 \%$ they identified 22 hits of which the top four were sequenced and confirmed in subsequent assays. Recently, Quartararo et al. ${ }^{10}$ demonstrated a synthesis of $10^{8}$ member peptide library and a screening strategy using affinity selection-mass spectrometry (AS-MS) methodology. ${ }^{11}$ In-solution affinity selection was combined with nanoliquid chromatography-tandem mass spectrometry peptide sequencing to identify the highest affinity binders. In this method, the target was immobilized onto magnetic beads and potential 
binders from the library were pulled down and sequenced by liquid chromatography-tandem mass spectrometry. The peptides in the libraries were 10 amino acids long with nine diversity positions. The library was designed to have a theoretical diversity of $2 \times 10^{11}$ but since it was made on $30 \mu \mathrm{m}$ TentaGel resin utilizing $2.9 \mathrm{~g}$ resin, it contained only a fraction of the library $-2 \times$ $10^{8}$ beads and peptides (with no compound redundancy in the library). The amino acids used for the library synthesis contained natural and non-natural amino acids, all with the standard scaffold of $\alpha$-amino acids.

Sequencing of $\alpha$-amino acid library hits on beads to identify peptides is routinely done by liquid chromatography with tandem mass spectrometry (LC-MS/MS) ${ }^{12}$ or Edman degradation on a protein sequencer, ${ }^{12 b}, 13$ which works well for short oligomer peptides but does not translate well to novel non- $\alpha$-amino acid backbone polymers. Many methods have been developed to improve the sequencing process such as introducing fluorescent dyes,${ }^{14}$ isotopic tags, ${ }^{15}$ DNA encoding, ${ }^{16}$ ladder-sequencing ${ }^{17}$ and chemical encoding methods. ${ }^{18}$ However, none of these sequencing methods are sensitive enough for libraries synthesized on beads of less than $20-30 \mu \mathrm{m}$ in diameter ${ }^{8 b}$ which contain $\sim 4$ picomoles of polymer / bead and for libraries with non- $\alpha$-amino acid backbone polymers. Historically ОВOC libraries have typically used bead sizes of $>90 \mu \mathrm{m}$ containing $>100$ picomoles of polymer per bead to ensure there is sufficient material for hit identification ${ }^{7}$ (Table S1). Large libraries of beads with these diameters are prohibitively expensive in materials costs to synthesize and screen, particularly for high molecular weight polymers, which is another reason why such chemical libraries sizes have been limited to date.

Here we present a novel platform that enables the production of large libraries of synthetic, sequence-defined non-natural polymers (NNPs) on the scale of $10^{7}-10^{9}$ members for megathroughput screening using a platform based on a Fiber-optic Array Scanning Technology 
(FAST ${ }^{\mathrm{TM}}$ ) that screens up to $\sim 5$ million polymers a minute. Furthermore, we describe a method for sequencing single bead hits down to $10 \mu \mathrm{m}$ in diameter with femtomole sensitivity. We demonstrate the platform's broad use in screening against five targets of biomedical interest to identify biologically relevant non-natural polymers with affinities in the nanomolar to subnanomolar range that can inhibit protein - protein interactions (PPIs) and protein - glycan interactions and have exceptional biological activity and stability.

\section{Results}

\section{FAST Screening Approach}

FAST was originally developed to identify rare circulating cancer cells in blood with high sensitivity and specificity (Figure 1). ${ }^{19}$ In this application, cells preincubated with fluorescently labeled cell surface markers are plated as a monolayer on $108 \times 76-\mathrm{mm}$ glass slides, which are then scanned by excitation with a $488 \mathrm{~nm}$ laser. Emitted fluorescence is collected through a fiber-optic bundle, and the collected light is passed through a bandpass filter and analyzed by a photomultiplier tube to measure emission at $520 \mathrm{~nm}$ (green) and $580 \mathrm{~nm}$ (red/orange) to eliminate true negatives due to autofluoresence (see below). Cartesian coordinates of fluorescently labeled objects are located on a pixel map, along with fluorescent intensity measurements at the two emission wavelengths. In this well-free assay format FAST can routinely identify the location of single rare cells in a milieu of 25 million white cells in a 1-minute scan with an $\sim 8$ m resolution. In optimizing the FAST system for bead screening the only major modification to the scanning process was the need to plate beads at a lower density than cells due to their propensity to aggregate and the need to extract them post-analysis for sequencing. Empirical optimization of bead plating density revealed that $10-\mu \mathrm{m}$ diameter TentaGel ${ }^{\mathrm{TM}}$ beads plated with a density of 5 million beads per plate 
gave a relatively well-dispersed monolayer enabling automated analysis and bead picking for down-stream processing. Similarly, $20-\mu \mathrm{m}$ beads were optimally plated at a density of 2.5 million beads per plate. Detection sensitivity was assessed by spiking biotin-labeled beads into a pool of underivatized beads and incubating with Alexa Fluor 555-labeled streptavidin for 1 hour before plating. The FAST-screening process gave a detection sensitivity of over 99.99\% (Table S2 and Figure S1).

In considering the application of FAST to bead based screening, two major problems complicate efficient fluorescence-based screening of TentaGel OBOC libraries: one problem is the autofluorescence of TentaGel beads leads to low signal-to-noise ratios and complicates the identification of hits. The FAST-screening approach uses several strategies to overcome problems due to bead autofluorescence based on optical properties of the TentaGel resin. Some key observations include the fact that TentaGel autofluorescence is highly significant in the FITC (fluorescein isothiocyanate) channel and the fluorescence intensity diminishes as its wavelength shift increases ${ }^{20}$; autofluorescence intensifies with increasing bead size; in our hands, functionalized beads with different chemistries have various levels of autofluorescence and in general are slightly higher than the autofluorescence of unfunctionalized beads. As mentioned above, the size of beads we use for our library construction in this study are 10-20 um in diameter (comparable to a mammalian-cell size) and the autofluorescence is significantly lower than the beads commonly used in other OBOC libraries (e.g., 90-300 um). Secondly, a more favorable fluorophore (Alexa-fluor 555 or CF555, yellow/orange) for target probes is used in conjunction with a wavelength comparison technique engineered in the FAST system to eliminate the effects of fluorescence from autofluorescing particles. ${ }^{21}$ The technique involves measuring emissions at two different wavelengths, one at the target emission wavelength $(580 \mathrm{~nm})$ and the other at $520 \mathrm{~nm}$, a wavelength intermediate between the target emission wavelength and the laser 
excitation (488 nm). Because autofluorescence is typically more intense at wavelengths closer to the excitation, the ratio of the intermediate wavelength intensity to the target wavelength intensity is greater than one for unlabeled beads while for labeled beads the ratio is less than one. A software filter uses this ratio to eliminate the autofluorescing beads. The software filter also screens for and eliminates fluoresce - positive objects originating from dye aggregates and bead fragments by filtering for object size and relative brightness. We set negative controls in parallel for each screen. Negative controls include fluorescence cutoffs determined from unfunctionalized naked TentaGel beads that have gone through the assay staining process with labelled probe, and a portion of the library beads taken through the assay staining protocol without the probe. As part of the comprehensive filter settings, the fluorescence intensity cutoff threshold is set to eliminate selection of autofluorescent beads due to the TentaGel or library background (filter threshold setting details are described in Table S6-11). With this filter, 99.8\% of the located objects on the glass slide can be eliminated as true negatives and are not selected for further analysis. With these filters the typical hit number from a FAST scan is around 100-400 identified as corresponding coordinate locations on the glass slide from a sample containing 2.5 million 20-um beads or 5 million 10-um beads.

The hits identified by the FAST primary scan are then automatically imaged and analyzed by high resolution Automated Digital Microscopy (ADM) on a CellCelector (ALS Automated Lab Solution $\mathrm{GmbH}$ ) using bright-field, target Alexa Fluor 555 (AF555) or CF555 and counter target AF647/Cy5 channels. Hit beads are QC/QA reviewed based on morphology and fluorescence staining data. Damaged beads, beads with irregular shape, size or staining pattern, and hit beads located within a large aggregate and impossible to exact are excluded. The Mean Fluorescence Intensity (MFI) is then measured for all hits that pass initial QC/QA. All "true positive" (TP) hits are ranked based on MFI intensity and/or ratio of selected channels and generally the top 50 beads from the 
initially 10- 400 FAST hits are selected and isolated for sequencing and hit confirmation by resynthesis and $K_{D}$ characterization.

Another problem with $\mathrm{OBOC}$ libraries is that during on-bead screening the signal strengths (e.g., fluorescence intensities) do not always correlate with the potency of the ligands on these beads. One of the contributing factors to this problem is that commercial resins typically used for library synthesis have high ligand loading (e.g., 90-um TentaGel resin with a loading capacity of 0.3 $\mathrm{mmol} / \mathrm{g}$ has a ligand density of $\sim 100 \mathrm{mM}$ ) which is necessary to provide a sufficient amount of material for subsequent hit identification, but may cause false positives and screening biases due to the unintended multidentate interaction with high ligand density on the beads ${ }^{20 c}$. Chen et al. have showed that the decrease of ligand concentration on the beads leads to a significantly reduced number of false positives due to the reduction of nonspecific binding caused by avidity effects ${ }^{22}$. We are similarly able to minimize avidity effects by the use of smaller beads with less ligand loading. This also allows us to use lower probe concentrations in screening. For every screen, the probes are pre-titrated to identify the minimum probe concentrations that achieve optimal signal-to-noise ratio and hit numbers (Titration and optimization details are described in Table S6-11). These strategies increase the probability of identifying the most active hit(s) while minimizing false positives.

\section{Self-Readable Polymers and the "Ptych" Approach}

We created a novel self-readable sequencing approach to polymer library design called the "ptych" (pronounced "tick") design. Figure 2a depicts a "tetraptych" (from Greek meaning "fourfold" from tetra, i.e., "four" and ptysso, i.e., "to fold"), which is a term used in the art world to describe a panel painting divided into four sections that can be folded to display a composite scene. In our application, a tetraptych is defined as a set of four monomers with folding 
properties that make up one diversity element in a longer sequence. The full sequence can then be formed by linking multiple ptychs together. Each tetraptych is selectively composed of monomers that enable diversity of physiochemical and structural properties at the individual ptych level. With this approach, polymer library scale and diversity can be built by choosing the number of ptychs in a linear sequence and the number of ptych variants at each position. The size of the ptych is also flexible. For example, two monomers define a diptych; four monomers define tetraptychs; and six monomers define hexaptychs and so forth.

By connecting ptychs via chemically cleavable linkers that can be cleaved under orthogonal conditions to those used in library synthesis and screening, the sequence of a ptych polymer can be directly read by mass spectrometry (MS). Figure 2 a depicts a general polymer design with three tetraptychs, each consisting of four diversity-building block monomers and a cleavable linker. Cleavage of the linker monomers yields an equimolar mixture of the three tetraptychs. As a preliminary proof-of-concept, we used the phenyl-acetamido-methylene (PAM) ${ }^{23}$ linker as a cleavable monomer building block. This monomer is normally used as a cleavable linker between a peptide and resin in Boc solid-phase peptide synthesis (SPPS). ${ }^{23}$ In our application, it provides an ester bond between ptychs that is stable to the Fmoc/tBu/Alloc protection strategy ${ }^{24}$ to build the polymers, but it can be readily cleaved using aqueous base such as ammonium hydroxide or sodium hydroxide. Figure $\mathbf{2} \mathbf{b}$ depicts a general polymer design with multiple tetraptychs, each consisting of a PAM linker and three diversity building-block monomers. Hydrolysis of the esters yields a mixture of the different tetraptychs.

The most important aspect of the ptych design is that building blocks can be selected so each ptych diversity element in a sequence has a unique molecular weight. As a result, each of the 
ptychs present in the mixture after cleavage can be identified by its mass using high-resolution LC-MS and an electrospray source on an LTQ-Orbitrap XL mass spectrometer that can detect molecular ions at the femtomole level. This provides three orders of magnitude more sensitivity than the MS fragmentation methods used in peptide and protein sequencing. ${ }^{25}$ Sequencing of ptych designed libraries is independent of the nature of the building blocks and virtually any chemical building block can be incorporated, whereas MS fragmentation sequencing is extremely dependent on the nature of the fragmentation patterns of the backbone chemical bonds of the building blocks and has largely been limited to $\alpha-$ amino acid peptide polymers. The $10-\mu \mathrm{m}$ diameter beads at $\sim 0.2 \mathrm{mmol} / \mathrm{g}$ loading typically carry $\sim 100 \mathrm{fmols}$ of compound that is readily detectable by high-resolution LC-MS (Table S1). Using ptych design sequencing, polymer libraries can be synthesized on much smaller diameter beads to create much larger libraries. Combined with the FAST platform, this enables screening and hit identification of much larger synthetic bead-based polymer libraries than has previously not been possible.

In a validation study to determine the reliability of sequences from individual beads, a set of 90 individual $10-\mu \mathrm{m}$ beads, each containing one of four possible unique sequences, were mixed and then picked from a plate and cleaved and sequenced. We were able to obtain the full correct sequence for 82 of the picked beads (91\%) (Tables S3a-d). There were no incorrect sequence assignments in any of the validation samples in which ptychs were detected. The samples that did not yield an identifiable sequence also did not yield any ptych assignments, indicating the beads were likely not deposited correctly in the vial or were otherwise lost during automated sample processing, which is a factor in microscale handling efficiency and hit confirmation rates.

Figure 3 summarizes the typical screening process of a polymer library using the FAST screening and ptych library design. Assay development involves a preliminary titration screen using varying 
concentrations of targets against the library and naked control beads (Tables S6-S10) to minimize the effects of autofluoresence as described above while maximizing signal to noise. Based on these results, target screening concentrations and the background MFI threshold are selected for optimum hit fluorescent signals relative to background (Table S11). The library is incubated with a fluorescently labeled target in 50\% Odyssey buffer and 0.5\% CHAPS blocking buffer to screen out non-specfic binding and is followed by a sequence of washes (Table S11). The beads are then plated as a monolayer on glass slides and FAST screened to identify positive hits defined as fluorescently labeled beads that indicates binding to the target (Figure 4a). The plate and the hit location data from FAST are transferred to an automated fluorescence microscope and picking robot (ALS CellCelector - Video S1) for preliminary hit quality control. Confirmed hit beads are individually transferred into vials and treated with cleavage solution to hydrolyze the backbone esters yielding a mixture of, in this case, tetraptychs which are sequenced by LCMS (Figure $\mathbf{4 b - c}$ ). The hit sequences are re-synthesized and purified by preparative high-performance LC (HPLC) for hit confirmation and further testing (Figure 4d).

A preliminary study of hits showed switching the backbone ester bonds to amides had only minor effects on measured binding affinities (Table S12), and as this greatly improves compound stability and simplifies hit resynthesis, all hits were prepared as the full backbone amide analogs. We measured resynthesized hit binding affinities for their respective targets using microscale thermophoresis (MST) (Figure 5c). Binding was confirmed in the majority of all backbone amide resynthesized hits with binding affinities in the nanomolar to sub-nanomolar range which indicated that switching out backbone esters for amide bonds generally has minimal effects on hit confirmation for these NNP designs. 


\section{Library Design and Screening Against Multiple Targets}

We synthesized two large non-natural polymer libraries labeled NNP1 and NNP2. NNP1 (Figure 4a) consists of six hexaptychs as the diversity elements in which each ptych was composed of four D-amino acids or glycine and an L-amino acid or glycine ester linked to a PAM linker. This produced polymers of 36 monomers in length with an average molecular weight of $\sim 5 \mathrm{kDa}$. Each ptych was designed to have one of 11 possible hexaptychs per diversity position (listed under Hexaptych 1, Hexaptych 2 etc. in Figure 4a), making a $11^{6}$ or an 1.77 million compound library. This corresponds to 66 hexaptychs, each of which was designed through a selection of monomers to give a range of physicochemical properties in each sequence position and a unique molecular weight for each hexaptych. Before synthesizing the library, we confirmed synthetic feasibility of each individual hexaptych as a reference to determine the retention time by LC-MS and facilitate the sequencing of hits (Table S4). We made 75 copies of the library on 20- $\mu \mathrm{m}$ diameter monosized amino TentaGel microsphere resin beads with a loading of 0.27 $\mathrm{mmol} / \mathrm{g}$, which required only $\sim 550 \mathrm{mg}$ of bead resin to produce.

Figure $\mathbf{4 b}$ shows the design for NNP2 that consists of nine tetraptychs constituting a total polymer length of 36 monomers. For each ptych in the sequence, there were 10 possible tetraptychs, constituting a total of 90 tetraptychs and creating a library of $10^{9}$ or one billion compounds. We

believe this is the largest bead-based synthetic sequence-defined NNP library reported to date. ${ }^{8 b}$ This library was constructed on $10-\mu \mathrm{m}$ beads and required only $1.5 \mathrm{~g}$ of resin for production of three copies (total of three billion beads). The individual ptychs in this library were also synthesized as controls and validated for sequencing (Table S5). After constructing the libraries, all side-chain protecting groups were removed and the libraries were screened against multiple biological targets. 
The two NNP libraries were constructed from two groups of building blocks. The first group included five pre-made fmoc-L-amino acid- (or Gly-) PAM esters: fmoc-L-Phe-PAM ester, fmoc-LAla-PAM ester, fmoc-L-Val-PAM ester, fmoc-L-Leu-PAM ester and fmoc-Gly-PAM ester. All the five amino acid-PAM-esters where commercially available with the boc- protecting group, which was simply converted to the fmoc form and was used in the library synthesis (see Supporting Information for synthesis). The second group of building blocks used in the library included 15 fmoc-protected D-amino acids (or Gly): Ala, Glu, Phe, Gly, His, Lys, Leu, Asn, Pro, Arg, Ser, Thr, Val, Trp and Tyr. NNP1 library was designed to have amino acid distribution closed to their average occurrence genome-wide (Table S15) (based on data from the UCSC Proteome Browser $\left.{ }^{26}\right)$. The library was designed in such a way that the amino acids will be distributed among the six hexaptychs as shown in Table S16. The design of NNP2 library was more unique with less resemblance to the amino acid distribution in the genome (Table S17), and also here the amino acids were distributed among the nine tetraptychs in the library (Table S18).

To demonstrate the speed and efficiency of the screening and sequencing process we screened five target proteins: K-Ras, ASGPR, IL6 and its receptor IL6R, and TNF $\alpha$. K-Ras is an oncology drug target that is mutated in $\sim 30 \%$ of cancers and is associated with uncontrolled cell proliferationparticularly in pancreatic and lung cancers with poor prognosis. ${ }^{27}$ ASGPR is the functional subunit of the asialoglycoprotein receptor (ASGPR) which is a C-type lectin glycan receptor predominantly found on the surface of liver hepatocytes and has been utilized as a mediator for liver-specific intracellular drug delivery of nucleic acid-based therapeutics. ${ }^{28} \mathrm{TNF} \alpha$, IL6, and soluble IL6 receptor (IL6R) are respectively cytokines and a cytokine receptor involved in immunological signaling processes and are well established immunotherapy targets. All are challenging targets for traditional small-molecule approaches and therefore represent interesting test cases for chemical NNP ligands. 
In the case of the K-Ras, IL6 and IL6R, we wanted to screen for protein - protein interaction inhibitors. For K-Ras we wanted to specifically block binding of the Ras binding domain to its downstream signaling partner Raf (Figure 5a-b), and for IL6 we wanted to block binding to its receptor IL6R and conversely in a separate screen find binders of IL6R that block IL6 binding. We labeled primary screening targets (Raf, IL6, IL6R) with dyes maximally excited at $~ 555 \mathrm{~nm}$ (AF555 or CF555) to identify binders in the FAST screen, and counter targets (Raf, IL6R, IL6) with dyes maximally excited at $647 \mathrm{~nm}$ wavelength (AF647 or CF647) which could be detected by ADM on the CellCellector. After FAST screening hit detection, we measured the MFI for each dye on each bead to prioritize the hits using both the overall brightness of the bead as a qualitative measure of binding affinity and the MFI ratio of the target to the counter target (Figure 5b,c).

K-Ras and ASGPR were screened against library NNP1. The library size of NNP1 is $1.77 \mathrm{M}$ members on $20-\mu \mathrm{m}$ beads, and it was screened at a 2.8-fold redundancy with $5 \mathrm{M}$ beads on two plates (2.5M beads per plate). Given the compound redundancy we could cross-validate hits via hit redundancy in the screen and found several hits with the same sequence (or 4-5 out of 6 hexaptychs in common, i.e., 67\%-83\% similarity within sequences). After FAST screening, we identified a preliminary hit list of $381 \mathrm{~K}$-Ras selective binding beads. Hit sequences in the K-Ras screen were pooled into 14 clusters, and the most prevalent sequences in each case were selected from each cluster. Similarly, 190 hit sequences from the ASGPR screen were grouped into 19 clusters and individual hits from each cluster were selected for hit confirmation by resynthesis and measurement of $K_{D}$ by MST (Table S13). Equilibrium $K_{D}$ binding affinities for KRas hits ranged from $18-180$ nM, and 0.22 - 330 nM for ASGPR (Figure 5d, Table S13).

With a library size of $1 \mathrm{~B}$ members on $10-\mu \mathrm{m}$ beads, library NNP2 would require 200 plates to screen the entire library at $5 \mathrm{M}$ beads per plate. With a custom industrial robotic high-throughput 
screening (HTS) suite, this would be fairly straightforward-the entire library could be FAST screened in $<10 \mathrm{hr}$. In this proof-of-concept study, we manually screened a 10 million portion of the library, corresponding to 2 screening plates against IL-6, IL6R and TNF $\alpha$. As with the K-Ras Raf screen, we performed the IL-6 screen using counter labeled IL-6R labeled to identify IL6 binding domain selective inhibitors. Similarly, we performed the screen against IL-6R using counter labeled IL-6. We selected and isolated the hits with the highest target to anti-target MFI ratios. For TNF $\alpha$, we were primarily interested in finding selective affinity agents and did not conduct a competition screen. Binding affinities ranged from 25-500 nM for IL6 , $0.6-330 \mathrm{nM}$ for IL6R and $0.3-270 \mathrm{nM}$ for TNF $\alpha$. The most potent hit in the NNP2 screen was a $\mathrm{K}_{\mathrm{D}}$ of $620 \mathrm{pM}$ against IL-6R.

Table S14 shows the hit rate broken down by screening and sequencing steps across the five targets. The average hit rate for beads identified by the FAST screen and passing QC/QA in ADM is $0.003 \%$ and ranged from 19 hit beads for IL 6 to 381 hits for K-Ras. The bead hit rate is to a large extent determined by the threshold cut identified in assay development to eliminate the effects of autofluorescence producing false negatives. This is also a reasonable hit rate in terms of the downstream processing effort for hit confirmation. Hit bead selection for automated picking from the screening plates depends primarily on how isolated the beads are from neighboring beads. In a number of cases hit beads are located in dense aggregates that make picking difficult to impossible without carrying over several other beads that will confound sequencing. In the five assays shown here on average allowed $72 \pm 44 \%$ of the hit beads to be picked. Of these on average $81 \pm 25 \%$ were successfully sequenced by LCMS. The factors affecting sequencing were successful transfer of the beads to the cleavage vial where failure results in no observable ptychs, or incomplete sequencing where individual ptychs failed to be identified in the LCMS. Sequencing 
however did not prove to be a major challenge and the high sequencing rate enabled hit confirmation by resynthesis without major optimization. Where we identified a large number of redundant hits such as for K-Ras, ASGPR and IL6R, sequences were clustered and the highest represented sequence in each cluster was selected for resynthesis. Over the five targets screened hit confirmation of resynthesized hits was $71 \pm 29 \%$ denoting a high true positive rate.

\section{Discovery of PPI Inhibitors and Receptor Mediated Intercellular Delivery Agents}

To demonstrate the biological significance of NNPs and their target selectivity, we focused on KRas and ASGPR functional biological activities. For K-Ras we investigated specific inhibition of KRas and Raf binding for a range of confirmed K-Ras hits. We tested this in an MST competition binding assay (Figure 6a). As a control we measured the K-Ras - Raf interaction alone for which an average $K_{D}$ values of $78 \mathrm{nM}$ was obtained from two technical runs. Then, we measured the same interaction with three different K-Ras lead hits (KRAS-1-4, $K_{D}=36 \mathrm{nM}$; KRAS-1-8, $K_{D}=44$ $\mathrm{nM}$; KRAS-1-13, $\mathrm{K}_{\mathrm{D}}=30 \mathrm{nM}$; at $1 \mu \mathrm{M}$ each), separately pre-incubated with $\mathrm{K}$-Ras at room temperature for 15 minutes. The three NNPs showed a range of inhibition activity from complete inhibition (KRAS-1-8), partial inhibition (KRAS-1-13), and no inhibition (KRAS-1-4). The MFI ratios of the target (K-Ras-CF555) to the counter target (Raf-AF647) for the hit beads corresponding to these hits was higher for KRAS-1-8 than that of the other two hit-beads (MFI ratios: KRAS-1-8 = 2.55; KRAS-1-4 $=1.89$ and KRAS-1-13 $=1.53$ ), suggesting that this could be a useful metric for functional inhibitors of PPIs from the primary competition screen.

ASGPR is a glycoprotein receptor, and all the published ligands are glycans that to date mimic the native substrates. ${ }^{29}$ To determine if NNP hits could specifically internalize into liver cells with high expression of ASGPR but not cells lacking ASGPR expression, we compared ASGPR-mediated uptake of two lead NNP hits, ASGPR-9-4 $\left(K_{D}=230 \mathrm{nM}\right)$ and ASGPR-9-6 $\left(K_{D}=34 \mathrm{nM}\right)$, in the HepG2 
human hepatocarcinoma (high expressing) and HEK293 (non-expressing) cell lines (Figure 6b). As a positive control we utilized the ASGPR trivalent ligand $\mathrm{N}$ - acetylgalactosamine (GaINAc) ${ }^{29 a}$ and a non-hit NNP from the same NNP1 library (KRAS-1-14) as a negative control. All compounds were labeled with fluorescein and analyzed by flow cytometry (see Methods for detailed procedures, and Supporting Information for synthesis). Internalization of the two NNP hits was significantly higher in HepG2 cells compared to in HEK293 cells, and significantly higher than uptake of the positive control GalNAc. The non-hit NNP negative control showed minimal uptake in either cell line. To further demonstrate ASGPR mediated cellular uptake, competitive cell uptake assays were performed utilizing the two NNP hits and the positive control GalNAc in HepG2 cells in the presence of asialofetuin, a naturally occurring serum protein ligand for ASGPR ${ }^{30}$ (Figure 6b). Cells were pre-incubated with two concentrations of asialofetuin, $(20 \mu \mathrm{M}$ and $60 \mu \mathrm{M})$ representing 67 and 200-fold excess compared with the test compound $(0.3 \mu \mathrm{M})$. The NNP hits and positive control's cellular uptake decreased with increasing concentrations of asialofetuin, and is mostly abolished with $60 \mu \mathrm{M}$ of asialofetuin. These results indicate that these ligands compete for the same receptor, and that uptake is ASGPR-mediated. Importantly these results for K-Ras and ASGPR demonstrate that the NNP hits found in screening are not non-selective binders but are capable of selectively binding to their target proteins in the presence of other selective proteinbinding partners, plasma media and cell membranes and are capable of eliciting functional biological responses.

\section{Biological Stability of NNP Hits}

To confirm the superiority in stability of these largely D-amino acid NNPs over peptides, we investigated the stability of an IL-6R hit from NNP2 to proteinase K and in human plasma. For the proteinase $\mathrm{K}$ stability assay, we compared the original hit with its fully L-amino acid variant. As 
expected, the L-amino acid variant was completely degraded within less than 2 hours in the presence of proteinase K (Figure 6c), whereas minimal degradation was observed for the NNP hit after an overnight incubation. Similar stability was observed in human plasma where the stability of the hit was compared to the natural peptide Angiotensin I. Angiotensin I was completely degraded within 4 hours in human plasma (Figure 6c), whereas NNP2 hits stayed largely intact even after overnight incubation.

\section{Conclusions}

Using the FAST screening platform and ptych design, we have demonstrated a mega-throughput screening and sequencing strategy for the discovery of potent and functional NNPs. The novel ability to screen at the femtomole scale on $10-\mu \mathrm{m}$ beads enables time and cost-effective screening with much larger chemical diversity than has previously been reported. In this proofof-concept study, we used commercially available amino acid building blocks and wellestablished solid phase chemistry to construct these first NNP libraries to validate the screening and sequencing methodology. Using the same approach, it is relatively straightforward to move into increasingly novel synthetic building blocks and coupling chemistry ${ }^{31}$ as well as different cleavable linkers enabling an unlimited access to polymer diversity through library synthesis and empirical screening. We have shown here that we can find low nanomolar to picomolar hits from primary screening and have used this to validate biological selectivity and activity in a range of molecular targets. We have shown biological functionality of the hits of two targets as representative use cases, which are the ability to disrupt PPI by inhibition of the K-Ras/Raf interaction, and protein-glycan interaction (PGI) in ASGPR-mediated cellular uptake and internalization. While we do not anticipate $\alpha$-amino acid NNPs will be passively permeable to cell membranes, our interest in screening for inhibitors of K-Ras which is an intracellular target was 
driven by recent breakthroughs in cell selective receptor mediated intracellular delivery of biologic molecules ${ }^{32}$ which could feasibly be used for delivery of NNP-like payloads. Utilizing a similar approach, we have also identified NNPs that could potentially be intracellular delivery agents by targeting ASGPR to identify receptor selective NPPs that not only bind ASGPR but are actively transported across cell membranes in a selective receptor mediated manner. The NNP hits identified here, without optimization, are more efficiently intracellularly transported than the previously reported molecular transport ligand trivalent GalNAc which is being used commercially for the delivery of nucleic acid drugs. ${ }^{28}$ This is particularly noteworthy as GalNAc and other reported ligands for ASGPR are glycans and we have demonstrated here that ASGPR can also bind and transport non-natural peptide-like ligands. Lastly, as observed by others, ${ }^{8 b}$ the primarily D-amino acid NNPs show unique stability against biological degradation.

Transition melt temperatures across all hits ranged from $39-65^{\circ} \mathrm{C}$ (data not shown), which is within the range of folded proteins of similar lengths (for example see ${ }^{33}$ ) and indicates that tertiary structure is probably important for the molecular interactions of these hits. As the diversity of synthetic polymers expands, 3D structures of hits by crystallography or nuclear magnetic resonance (NMR) will most likely identify templates for novel secondary and tertiary structural motifs that can be rapidly refined by building focused libraries for secondary screening. As structural motifs become better understood, individual ptychs can be engineered to promote intra- and inter-molecular recognition to stabilize structure and maximize affinity. We used a regular repeating ptych design for libraries described here, but more elaborate designs that use different numbers and types of monomers in ptych positions are possible. These will provide further chemical diversity for primary screening and strategies that allow optimization of hits. 
In summary, we have demonstrated a method for stepping outside of the bounds of natural polymers and moving into a new field of designer polymers with completely new structure and function through empirical screening. The application area of this platform is vastly broad and includes therapeutics for drug discovery, affinity reagents for sensors and diagnostics, and reagents for catalysis.

\section{Methods}

\section{Synthesis of Libraries NNP1 and NNP2.}

All libraries were synthesized using "one-bead-one-compound" and "mix-and-split" methods of solid-phase synthesis on TentaGel$^{\circledR}$ amine $10 \mu \mathrm{m}$ or $20 \mu \mathrm{m}$ resin. Library NNP1 was synthesized on $554 \mathrm{mg} 20 \mu \mathrm{m}$ TentaGel ${ }^{\circledR} \mathrm{M} \mathrm{NH}_{2}(0.27 \mathrm{mmol} / \mathrm{g}$ amine loading) with theoretical diversity of $1.77 \times 10^{6}$ and 75 copies (i.e., $1.33 \times 10^{8}$ beads). Library NNP2 was synthesized on $1.5 \mathrm{~g} 10 \mu \mathrm{m}$ TentaGel ${ }^{\circledR} \mathrm{M} \mathrm{NH}_{2}\left(0.25 \mathrm{mmol} / \mathrm{g}\right.$ amine loading) with theoretical diversity of $1 \times 10^{9}$ and 3 copies (i.e., $3 \times 10^{9}$ beads).

For the synthesis of library NNP1 the beads were swollen in DCM for 1 hour. Then the DCM was drained, and the beads were suspended in DMF and were divided evenly by pipet between 11 plastic fritted syringes placed on a manifold. Then 11 different hexaptychs were constructed on the beads, a different hexaptych in each fritted syringe, by coupling first an L-amino acid-PAM ester followed by the coupling of four more D- amino acids, according to the library design in Figure 4a. The beads were then mixed and split evenly again between the 11 plastic fritted syringes, and the synthesis was carried on in the same manner with the next hexaptychs, until all the six hexaptychs were constructed. 
For the synthesis of library NNP2, after swelling the beads in DCM for 1 hour, the DCM was drained, and the beads were suspended in DMF. Then, the beads were divided evenly by pipet between 10 plastic fritted syringes placed on a manifold. Then 10 different tetraptychs were constructed on the beads, a different tetraptych in each fritted syringe, by coupling first an Lamino acid-PAM ester followed by the coupling of two more D- amino acids, according to the library design in Figure 4b. The beads were then mixed and split evenly again between the 10 plastic fritted syringes, and the synthesis was carried on in the same manner with the next tetraptychs, until all the nine tetraptychs were constructed.

Coupling conditions for fmoc-L-amino acid-PAM esters in the library synthesis: 3.5 eq. fmoc-Lamino acid-PAM ester was dissolved in a solution of 0.5 M HATU in NMP (3.18 eq. HATU). Then DIEA (10 eq.) was added to this mixture to activate the amino acid for 30 seconds, and the solution was added to the resin and reacted for 30 minutes. After completion of the coupling reaction (confirmed by ninhydrin test), the resin was drained and washed with DMF ( $3 \times 5 \mathrm{~mL})$.

Coupling conditions for fmoc-D-amino acids: 5.5 eq. fmoc-D-amino acid was dissolved in a solution of $0.5 \mathrm{M} \mathrm{HATU}$ in NMP (5 eq. HATU). Then DIEA (10 eq.) was added to this mixture to activate the amino acid for 30 seconds, and the solution was added to the resin and reacted for 30 minutes. After completion of the coupling reaction (confirmed by ninhydrin test), the resin was drained and washed with DMF $(3 \times 5 \mathrm{~mL})$.

Fmoc deprotection: Fmoc deprotection was performed by the addition of 25\% 4-methylpiperidine in DMF $(5 \mathrm{~mL})$ to the resin $(1 \times 5 \mathrm{~min}+1 \times 10 \mathrm{~min})$, followed by draining and washing the resin with DMF $(5 \times 5 \mathrm{~mL})$.

Side-chain deprotection: At the end of the library's construction, after the last fmoc deprotection, all the library beads were mixed into one fritted syringe and the side-chain protecting groups 
were removed with a solution of $95 \%(\mathrm{v} / \mathrm{v})$ TFA, $2.5 \%(\mathrm{v} / \mathrm{v})$ water and $2.5 \%(\mathrm{v} / \mathrm{v})$ triisopropylsilane ( $1 \mathrm{~mL}$ of cleavage solution per $10 \mathrm{mg}$ of resin) for 2 hours. Then the TFA cocktail was drained, and the resin was thoroughly washed with DCM, DMF, DCM and $\mathrm{MeOH}(3 \times 10 \mathrm{~mL}$ of each solvent) and was ready for the screening process.

\section{Activation of K-Ras by GTP Loading for the Screen and Binding Assays.}

To activate K-Ras for binding NNP or Raf, the K-Ras protein had to be loaded with GTP. Loading was performed according to the following protocol: The $200 \mu \mathrm{M}$ stock solution of the target protein was diluted to $10 \mu \mathrm{M}$ in $20 \mathrm{mM}$ HEPES pH 8.0, $150 \mathrm{mM} \mathrm{NaCl}, 10 \mathrm{mM} \mathrm{MgCl}$, $1 \mathrm{mM} \mathrm{TCEP}$, 0.05\% Tween-20 (total volume $110 \mu \mathrm{L}$ ). $10 \mu \mathrm{L}$ were set aside for later labeling quality control. EDTA pH 8.0 (stock concentration $10 \mathrm{mM}$ ) was added to the protein solution to a final concentration of $80 \mu \mathrm{M}$. GTP (stock concentration $50 \mathrm{mM}$ ) was added to the protein solution to a final concentration of $750 \mu \mathrm{M}$. The solution was incubated at $30^{\circ} \mathrm{C}$ for 2 hours (PCR tube) and then placed on ice for 2 minutes. $\mathrm{MgCl}_{2}$ was added to the protein solution to a final concentration of $100 \mathrm{mM}$. The resulting protein solution was buffer exchanged into the buffer required in the labeling kit for labeling. This procedure was used before the screen, the MST analysis and the KRas/Raf inhibition assay. All the other target molecules (TNF $\alpha$, IL-6, IL-6R and ASGPR) were used as received without any additional treatment.

\section{Screening of OBOC Libraries on FAST.}

FAST screening assay were specifically optimized for each target in terms of probe concentration, blocking and washing stringency etc. The probe binding to the NNP1 and NNP2 library beads was performed in tubes. Typically, the library or control beads were hydrated in the buffer (1\% PEG, $50 \mathrm{mM}$ Tris, $\mathrm{pH} 7.5,25 \%$ Odyssey blocking buffer PBS) for $30 \mathrm{~min}$ at RT with vortex followed by 1 
min of sonication to break apart the large bead clumps. Beads were then centrifuged down, and the bead pallets were washed $2 X$ with Odyssey/PBS buffer, the bead suspension was further filtered through a 30-um size cell strainer to remove bead aggregates. The concentration of the hydrated beads was determined based on bead counting using a hemocytometer. Aliquots of the bead suspension with the required number of beads then were centrifuged down and resuspended in blocking buffer ( $100 \%$ Odyssey, $0.5 \%$ Chaps, $200 \mathrm{mM} \mathrm{NaCl}$ in PBS) and incubated overnight at RT with gentle rotating. After blocking, the beads were pelleted and resuspended in $100 \%$ Odyssey buffer and then mixed at a 1:1 volume ratio) with the CF555 or AF555 conjugated probe that was diluted in the pre-binding buffer ( $1 \%$ Chaps, $400 \mathrm{mM} \mathrm{NaCl}, 2 \mathrm{mM}$ TCEP, in PBS) to $2 \mathrm{X}$ final working concentration. The probe/library bead mixture were incubated for 1 hour at RT with gentle rotation to allow probe bind to the library beads. After incubation, the beads were palleted and the unbound probes were aspirated, followed by 3 washes with $10 \mathrm{ml}$ of wash buffer (0.5\% Chaps, $200 \mathrm{mM} \mathrm{NaCl}, 1 \mathrm{mM}$ TCEP in PBS), 5 mins/time and additional 2 washes with $10 \mathrm{ml}$ of $0.5 \%$ Chaps /PBS. After the last centrifugation, the buffer was aspirated but left final $\sim 500 \mu l$ to resuspend the beads in this residual buffer and sonicate them for 30 second to dissociated newly formed bead clumps. Then $1.5 \mathrm{ml}$ prepared $0.3 \%$ low melting agarose (LMT) that were kept in $37 \mathrm{C}$ water-bath before use were added to into the re-sonicated beads to make the bead/soft agar suspension.

Beads in the LMT suspension were then transferred and evenly plated onto the FAST slide (the screening plates) and then the slides were placed on cold tray to accelerate the curing and immobilization of the beads. Following the gel formation, a layer of mounting medium (e.g. 500 $\mu$ l Live-Cell medium) was gently placed on top of the gel to keep the beads from rapid drying or photo-quenching of the fluorescence. The sample slides (plates) were scanned and analyzed 
using the FAST system. The FAST analysis generates a bead hit list, where each bead is quantified by a MFI measurement.

\section{Bead Analysis and Picking Using ALS CellCelector.}

The beads with MFI values above a threshold determined by the "no probe" control condition were identified and then coordinate list of the hits were transferred to the CellCelector for Automated Digital Microscopy (ADM). This imaging analyzes the hits with multiple channels at higher resolution. Images of the hit beads were then QC/QA reviewed based on the morphology and fluorescence staining, and fluorescence of selected channels were quantified to rank the top hits for isolation, then each selected single hit-beads was isolated with CellCelector individually into the HPLC vials in $\mathrm{ddH}_{2} \mathrm{O}$ for MS based sequencing.

\section{Processing and Sequence Analysis of Picked Beads.}

Beads were deposited directly into glass autosampler vials containing deionized water. The vials were inserted into deep-well 96-well plates and dried in a vacuum centrifugal concentrator (GeneVac II Plus) at $40{ }^{\circ} \mathrm{C}$. To hydrolyze the inter-ptych ester linkages $50 \mu \mathrm{L}$ of $7 \%$ aqueous ammonium hydroxide or $150 \mathrm{mM} \mathrm{NaOH}$ was added, and the samples incubated at $37^{\circ} \mathrm{C}$ for $6 \mathrm{~h}$ and then evaporated under vacuum in the centrifugal concentrator. The samples were then prepared for analysis by adding $50 \mu \mathrm{L}$ of $5 \%$ acetonitrile in water with $0.1 \%$ formic acid. and analyzed by capillary reversed-phase gradient LC-MS/MS using an Agilent capillary HPLC pump and CTC Analytics autosampler coupled to an LTQ-Orbitrap mass spectrometry system. Expected masses of hydrolysis products were loaded into an inclusion list for targeted MS/MS when detected above threshold in a high resolution Orbitrap scan. Data analysis used both MS and MS/MS data to assign high confidence hits for assembling sequences for the hits. 


\section{Hit Re-synthesis.}

Solid phase NNP synthesis: Hits were synthesized on ChemMatrix Rink amide resin (loading 0.5 $\mathrm{mmol} / \mathrm{g}$, typical scale: $30 \mathrm{mg}, 0.015 \mathrm{mmol}$ ) by an automated peptide synthesizer (Biotage Syro I) using standard fmoc-based amide coupling conditions with DIC/Oxyma as the coupling reagents. Fmoc protected L-amino acid-PAM esters used in the library synthesis were replaced here by two separate residues: fmoc-L-amino acid and fmoc-PAM. This was in order to avoid having ester linkage (but rather a standard amide linkage) in the synthesized hits, for stability purposes. Synthesis was performed using the following protocol: ChemMatrix Rink amide resin was swollen in DCM for $1 \mathrm{~h}$, drained, washed with DMF and placed on the peptide synthesizer for constructing the full sequence. Fmoc deprotection was performed by the addition of $25 \% 4$-methyl-piperidine in $\mathrm{DMF}(1.2 \mathrm{~mL})$ to the resin $(1 \times 5 \mathrm{~min}+1 \times 10 \mathrm{~min})$, followed by draining and washing the resin with DMF ( $5 \times 1.2 \mathrm{~mL})$. Couplings were performed by adding $250 \mu \mathrm{L}$ of NMP to the resin followed by $90 \mu \mathrm{L}$ of $0.5 \mathrm{M}$ fmoc-protected amino acids (or fmoc-PAM) in DMF (3 eq., $0.045 \mathrm{mmol}$ ), $90 \mu \mathrm{L}$ of $0.5 \mathrm{M}$ Oxyma in DMF (3 eq., $0.045 \mathrm{mmol}$ ) and $90 \mu \mathrm{L}$ of $0.5 \mathrm{M}$ DIC in DMF (3 eq., $0.045 \mathrm{mmol}$ ). The resin-mixture was allowed to react for 15 minutes at $60 \mathrm{oC}$ and was then drained, washed with DMF $(3 \times 1.2 \mathrm{~mL})$ and treated again with the same coupling conditions for double coupling. At the end of the double coupling, the fmoc was deprotected and these synthesis cycles were repeated on the peptide synthesizer until all the residues were constructed onto the resin. After the last fmoc-deprotection, the resin-NNP was taken out of the peptide synthesizer for manual fluorescein incorporation.

Incorporation of fluorescein: fluorescein was incorporated on the $\mathrm{N}$-terminus of all the resynthesized hits. $21.3 \mathrm{mg}$ NHS-fluorescein (3 eq., $0.045 \mathrm{mmol}$ ) was dissolved in $300 \mu \mathrm{L}$ DMF and was added to the resin-NNP. The resin-mixture was allowed to react for $3 \mathrm{~h}$ and was monitored 
by ninhydrin test. Upon completion, the resin was drained and washed thoroughly with DMF (3 $\times 5 \mathrm{~mL})$ and $\mathrm{DCM}(3 \times 5 \mathrm{~mL})$ and was dried before cleavage.

NNP cleavage: Cleavage from solid support and side-chain deprotection were performed by treatment of resin-NNP with a solution of $95 \%(\mathrm{v} / \mathrm{v})$ TFA, $2.5 \%(\mathrm{v} / \mathrm{v})$ water and $2.5 \%(\mathrm{v} / \mathrm{v})$ triisopropylsilane ( $3 \mathrm{~mL}$ of cleavage solution per $30 \mathrm{mg}$ of resin) for 2 hours. TFA was then evaporated on the SpeedVac (Thermo Scientific Savant SpeedVac Concentrator) until the solution volume reached to $\sim 1 \mathrm{~mL}$. The crude NNP was then precipitated and triturated with chilled diethyl ether $(x 3)$ and was then purified by preparative LC-MS as described above (See Supporting Information for LC-MS analysis of representative crude NNP hits).

\section{Hit Characterization.}

Hit binding affinity to various targets were determined using Microscale Thermophoresis (MST). MST experiments were performed on a Monolith NT.115pico (NanoTemper Technologies GmbH, Munich, Germany). Measurements were performed at room temperature, in triplicate with incubation periods of 15, 30 and 45 minutes. Binding affinities were obtained from a 16 point, two fold dilutions series with ligand starting concentration at $1 \mu \mathrm{M}$ and target concentration at 5 nM. Targets were labeled using Nanotemper Monolith $2^{\text {nd }}$ Generation Protein Labeling Kits. REDMALEIMIDE (Maleimide-647-dye) labeling kit was used for K-Ras and RED-NHS (NHS-647-dye) labeling kit was used for IL-6, IL-6R, TNF $\alpha$ and ASGPR. The buffer for the ASGPR contained $20 \mathrm{mM}$ HEPES pH 7.4, $150 \mathrm{mM} \mathrm{NaCl}, 10 \mathrm{mM} \mathrm{MgCl}$, $2 \mathrm{mM} \mathrm{CaCl}_{2}, 0.05 \%$ Pluronic F-127, and $1 \mathrm{mM}$ DTT; for IL-6 contained $20 \mathrm{mM}$ HEPES pH 7.4, $150 \mathrm{mM} \mathrm{KCl}, 10 \mathrm{mM} \mathrm{MgCl}_{2}$, and 0.1\% Pluronic F-127; for the soluble IL-6 receptor $20 \mathrm{mM}$ HEPES pH 7.4, $150 \mathrm{mM} \mathrm{NaCl}, 10 \mathrm{mM} \mathrm{MgCl}$, and 0.05\% Tween20; for K-Ras 20 mM HEPES pH 7.4, 150 mM NaCl, 10 mM MgCl , 0.05\% Tween-20 and 1 mM DTT; 
for TNF $\alpha 10 \mathrm{mM}$ HEPES pH 7.4, $150 \mathrm{mM} \mathrm{NaCl}, 10 \mathrm{mM} \mathrm{MgCl}$, 0.05\% Polysorbate-20. Triplicate data was analyzed using MO.AffinityAnalysis software (NanoTemper Technologies GmbH).

\section{K-Ras/Raf Inhibition Assay.}

Interaction between the target protein K-Ras and the ligand protein Raf was investigated using a MicroScale Thermophoresis (HTS-MST) assay in the absence and presence of three NNP hits: KRAS-1-4, KRAS-1-8 and KRAS-1-13. Loading of K-Ras with the GTP was performed according to the protocol detailed above (Activation of K-Ras by GTP loading). After the GTP loading the resulting protein solution was buffer exchanged into $100 \mathrm{mM} \mathrm{HEPES} \mathrm{pH} \mathrm{6.5,} 5 \mathrm{mM} \mathrm{MgCl}$, $50 \mathrm{mM}$ $\mathrm{NaCl}, 1 \mathrm{mM}$ TCEP. The resulting concentration of the target protein was $8.9 \mu \mathrm{M}$, which was used for Maleimide-647-dye labeling. For the interaction between K-Ras and Raf with no NNP present two technical runs with the same samples were performed between GTP-loaded K-Ras and Raf in the same buffer conditions that were used to test the interaction between K-Ras and the NNP hits: $20 \mathrm{mM}$ HEPES pH 7.4, $150 \mathrm{mM} \mathrm{NaCl}, 10 \mathrm{mM} \mathrm{MgCl2,} 1 \mathrm{mM}$ DTT, 0.05\% Tween-20. For the interaction between K-Ras and Raf in the presence of NNPs the labeled target protein K-Ras was diluted to $10 \mathrm{nM}$ in assay buffer containing $2 \mu \mathrm{M}$ NNP and incubated at room temperature for 15 min. This solution was then mixed with the ligand protein Raf serial dilution 1:1 to yield the final assay samples with $5 \mathrm{nM}$ target protein and $1 \mu \mathrm{M}$ NNP.

\section{Cell Culture for ASGPR Uptake Assay.}

The HEK293T (human embryonic kidney cells) and human hepatoma HepG2 cells were grown according to the protocols provided by the American Type Culture Collection (ATCC). When cells reached cell were seeded at $\sim 1.5 \times 10^{5}$ cells/well in 24 -well culture plate for the uptake assay. 
After at least 16 hours of culture to allow cell attach and equilibrate, the compound treatment was set up for the uptake assay.

\section{ASGRPR Uptake Assay.}

The fluorescein-labeled NNPs or fluorescein-labeled N-acetylgalactosamine (GalNAc) recognizing ASGRPR were added to wells at indicated concentrations and incubated for 2 hours. Two plates were prepared for each treatment condition, one serving as $4{ }^{\circ} \mathrm{C}$ no internalization control that was kept on ice during incubation, while the second plate was incubated at $37 \circ \mathrm{C}$ to allow for energy dependent internalization. Following the incubation period, all plates were placed on ice and washed three times with ice-cold PBS/3\% BSA/2 mM EDTA and then lifted with trypsin. Cells were transferred to a 96-well round bottom plates in FACS buffer. Cells were then analyzed by flowcytometry using LSR-II with HTS sampler (BD Biosciences, San Jose, CA, USA). Data (mean fluorescence intensities) and further analyzed using Flowjo software (BD Biosciences, San Jose, CA, USA). The internalized fraction was expressed as the difference between the corresponding $4 \circ \mathrm{C}$ and $37{ }^{\circ} \mathrm{C}$ MFIs as previously described. ${ }^{28}$

For the competitive uptake assay with the natural ligand of ASGPR (asialofetuin), cells were preincubated with $20 \mu \mathrm{M}$ and $60 \mu \mathrm{M}$ asialofetuin on ice or at $37 \circ \mathrm{C}$ for 1.5 hours, followed by the treatment with compounds for additional 2 hours before flow cytometric analysis as described above. The reduction of the uptake under asialofetuin competition was expressed by the percentage against the same treatment condition without asialofetuin. ${ }^{34}$

\section{Stability Assays.}

For proteinase $\mathrm{K}$ stability, solutions of each tested compound (200 $\mu \mathrm{M})$ in 10\% DMSO and $20 \mathrm{mM}$ Tris $\mathrm{HCl}$ at $\mathrm{pH} 8$ were prepared. Proteinase $\mathrm{K}$ was added to a final concentration of $100 \mu \mathrm{g} / \mathrm{mL}$ 
and $100 \mu \mathrm{M}$ of the tested compound in $5 \% \mathrm{DMSO}$ and $10 \mathrm{mM}$ Tris $\mathrm{HCl}$ at $\mathrm{pH}$. The solutions were incubated at $37^{\circ} \mathrm{C}$ and aliquots after $0 \mathrm{~h}, 1.5 \mathrm{~h}$ and $16 \mathrm{~h}$ were analyzed by LC-MS.

For human plasma stability, lyophilized human plasma was reconstituted in sterile water for injection, aliquoted into $200 \mathrm{uL}$ aliquots and stored frozen at $-80^{\circ} \mathrm{C}$ prior to the stability studies. For the stability studies three aliquots per NNP were thawed at room temperature. An additional set of three aliquots for a positive control peptide (Angiotensin I) were also thawed. The incubations for the plasma stability were initiated by mixing $2 \mathrm{uL}$ of a $2 \mathrm{mM}$ stock solution of NNP IL6R-87-8 or positive control in DMSO with the $200 \mathrm{uL}$ thawed plasma aliquot. After briefly vortex-mixing, $50 \mathrm{uL}$ zero-time-point samples were removed, mixed with $50 \mathrm{uL}$ of water and $400 \mathrm{uL}$ of acetonitrile and frozen on dry ice until all time point samples had been collected. The samples were incubated at $37^{\circ} \mathrm{C}$ with samples removed and water/acetonitrile added at 1 $\mathrm{h}, 3 \mathrm{~h}$ and $17 \mathrm{~h}$. Proteins were precipitated by centrifuging the samples at $17,000 \mathrm{G}, 4{ }^{\circ} \mathrm{C}$ for 1 h. The supernatants were removed and concentrated in a centrifugal vacuum concentrator (GeneVac Genie II) at $45{ }^{\circ} \mathrm{C}$ until the volume had been reduced to $\sim 60 \mathrm{uL}$. The samples were then diluted with 95\% water 5\% Acetonitrile and 0.1\% Formic Acid to volume of $200 \mathrm{uL}, 2 \mathrm{uL}$ of an internal standard peptide (Val5-Angiotensin I, Sigma) were added prior to samples analysis by LC-MS on the LTQ-Orbitrap XL system described above.

\section{Acknowledgements:}

This work was supported by the DARPA Fold F(X) program N66001-14-C-4059; (Distribution Statement "A" - Approved for Public Release, Distribution Unlimited. The views, opinions, and/or findings expressed are those of the authors and should not be interpreted as 
representing the official views or policies of the Department of Defense or the U.S.

Government.) We also thank SRI for internal R\&D support as well as experimental guidance from our colleagues Kathlynn Brown and Michael McGuire. The reference trivalent GaINAc ligand for ASGPR was kindly provided by lonis Pharmaceuticals, Inc. The K-Ras / Raf competition assay was performed by 2 bind $\mathrm{GmbH}$ (Germany).

\section{Author Contributions:}

NC conceived of the concepts presented here with support from PBM and MAS. MAS, TS, XL, PB, $\mathrm{JS}, \mathrm{CR}, \mathrm{AL}, \mathrm{ATO}, \mathrm{KY}$ and $\mathrm{LS}$ ran the experiments. MAS, PBM and NC wrote the manuscript.

\section{Competing Interests:}

The authors state no competing interests.

Additional Information 


\section{References}

1. (a) Barbas, C. F., 3rd; Kang, A. S.; Lerner, R. A.; Benkovic, S. J., Assembly of combinatorial antibody libraries on phage surfaces: the gene III site. Proceedings of the National Academy of Sciences of the United States of America 1991, 88 (18), 7978-82; (b) Nixon, A. E.; Sexton, D. J.; Ladner, R. C., Drugs derived from phage display: from candidate identification to clinical practice. mAbs 2014, 6 (1), 73-85; (c) Maeda, Y.; Javid, N.; Duncan, K.; Birchall, L.; Gibson, K. F.; Cannon, D.; Kanetsuki, Y.; Knapp, C.; Tuttle, T.; Ulijn, R. V.; Matsui, H., Discovery of catalytic phages by biocatalytic self-assembly. J Am Chem Soc 2014, 136 (45), 15893-6; (d) Seker, U. O.; Demir, H. V., Material binding peptides for nanotechnology. Molecules 2011, 16 (2), 1426-51.

2. (a) Langan, R. A.; Boyken, S. E.; Ng, A. H.; Samson, J. A.; Dods, G.; Westbrook, A. M.; Nguyen, T. H.; Lajoie, M. J.; Chen, Z.; Berger, S.; Mulligan, V. K.; Dueber, J. E.; Novak, W. R. P.; ElSamad, H.; Baker, D., De novo design of bioactive protein switches. Nature 2019, 572 (7768), 205210; (b) Huang, P. S.; Boyken, S. E.; Baker, D., The coming of age of de novo protein design. Nature 2016, 537 (7620), 320-7; (c) Wachsmuth, M.; Findeiß, S.; Weissheimer, N.; Stadler, P. F.; Mörl, M., De novo design of a synthetic riboswitch that regulates transcription termination. Nucleic Acids Res 2013, 41 (4), 2541-51.

3. (a) Hill, D. J.; Mio, M. J.; Prince, R. B.; Hughes, T. S.; Moore, J. S., A field guide to foldamers. Chemical reviews 2001, 101 (12), 3893-4012; (b) Zhao, Y.; Moore, J. S., Foldamers Based on Solvophobic Effects. In Foldamers, 2007; pp 75-108.

4. (a) Gellman, S. H., Foldamers: A Manifesto. Acc. Chem. Res. 1998, 31 (4), 173-180; (b) Chaput, J. C.; Herdewijn, P., What Is XNA? Angewandte Chemie (International ed. in English) 2019, 58 (34), 11570-11572; (c) Al Ouahabi, A.; Charles, L.; Lutz, J.-F., Synthesis of Non-Natural Sequence-Encoded Polymers Using Phosphoramidite Chemistry. Journal of the American Chemical Society 2015, 137 (16), 5629-5635; (d) Chen, Z.; Lichtor, P. A.; Berliner, A. P.; Chen, J. C.; Liu, D. R., Evolution of sequence-defined highly functionalized nucleic acid polymers. Nat. Chem. 2018, 10 (4), 420-427; (e) Kodadek, T., Synthetic receptors with antibody-like binding affinities. Curr. Opin. Chem. Biol. 2010, 14 (6), 713-20; (f) Badi, N.; Lutz, J.-F., Sequence control in polymer synthesis. Chem. Soc. Rev. 2009, 38 (12), 3383-3390; (g) Chan-Seng, D.; Lutz, J.-F., Solid-Phase Synthesis as a Tool for the Preparation of Sequence-Defined Oligomers Based on Natural Amino Acids and Synthetic Building Blocks. In Sequence-Controlled Polymers: Synthesis, Self-Assembly, and Properties, American Chemical Society: 2014; Vol. 1170, pp 103-116. 
5. Lam, K. S.; Salmon, S. E.; Hersh, E. M.; Hruby, V. J.; Kazmierski, W. M.; Knapp, R. J., A new type of synthetic peptide library for identifying ligand-binding activity. Nature 1991, 354 (6348), 82-84.

6. Komnatnyy, V. V.; Nielsen, T. E.; Qvortrup, K., Bead-based screening in chemical biology and drug discovery. Chemical Communications 2018, 54 (50), 6759-6771.

7. Lam, K. S.; Lebl, M.; Krchňák, V., The “One-Bead-One-Compound” Combinatorial Library Method. Chemical Reviews 1997, 97 (2), 411-448.

8. (a) Sklar, L. A.; Carter, M. B.; Edwards, B. S., Flow cytometry for drug discovery, receptor pharmacology and high-throughput screening. Current opinion in pharmacology 2007, 7 (5), 52734; (b) Gates, Z. P.; Vinogradov, A. A.; Quartararo, A. J.; Bandyopadhyay, A.; Choo, Z. N.; Evans, E. D.; Halloran, K. H.; Mijalis, A. J.; Mong, S. K.; Simon, M. D.; Standley, E. A.; Styduhar, E. D.; Tasker, S. Z.; Touti, F.; Weber, J. M.; Wilson, J. L.; Jamison, T. F.; Pentelute, B. L., Xenoprotein engineering via synthetic libraries. Proceedings of the National Academy of Sciences of the United States of America 2018, 115 (23), E5298-e5306.

9. Carney, R. P.; Thillier, Y.; Kiss, Z.; Sahabi, A.; Heleno Campos, J. C.; Knudson, A.; Liu, R.; Olivos, D.; Saunders, M.; Tian, L.; Lam, K. S., Combinatorial Library Screening with Liposomes for Discovery of Membrane Active Peptides. ACS combinatorial science 2017, 19 (5), 299-307.

10. Quartararo, A. J.; Gates, Z. P.; Somsen, B. A.; Hartrampf, N.; Ye, X.; Shimada, A.; Kajihara, Y.; Ottmann, C.; Pentelute, B. L., Ultra-large chemical libraries for the discovery of high-affinity peptide binders. Nature Communications 2020, 11 (1), 3183.

11. (a) Zuckermann, R. N.; Kerr, J. M.; Siani, M. A.; Banville, S. C.; Santi, D. V., Identification of highest-affinity ligands by affinity selection from equimolar peptide mixtures generated by robotic synthesis. Proc Natl Acad Sci U S A 1992, 89 (10), 4505-9; (b) Dunayevskiy, Y. M.; Lai, J.-J.; Quinn, C.; Talley, F.; Vouros, P., Mass spectrometric identification of ligands selected from combinatorial libraries using gel filtration. Rapid Communications in Mass Spectrometry 1997, 11 (11), 1178-1184; (c) Kaur, S.; McGuire, L.; Tang, D.; Dollinger, G.; Huebner, V., Affinity selection and mass spectrometry-based strategies to identify lead compounds in combinatorial libraries. $J$ Protein Chem 1997, 16 (5), 505-11; (d) van Breemen, R. B.; Huang, C.-R.; Nikolic, D.; Woodbury, C. P.; Zhao, Y.-Z.; Venton, D. L., Pulsed Ultrafiltration Mass Spectrometry: A New Method for Screening Combinatorial Libraries. Analytical Chemistry 1997, 69 (11), 2159-2164; (e) Maaty, W. 
S.; Weis, D. D., Label-Free, In-Solution Screening of Peptide Libraries for Binding to Protein Targets Using Hydrogen Exchange Mass Spectrometry. J Am Chem Soc 2016, 138 (4), 1335-43.

12. (a) Aebersold, R.; Mann, M., Mass spectrometry-based proteomics. Nature 2003, 422 (6928), 198-207; (b) Thakkar, A.; Cohen, A. S.; Connolly, M. D.; Zuckermann, R. N.; Pei, D., Highthroughput sequencing of peptoids and peptide-peptoid hybrids by partial edman degradation and mass spectrometry. Journal of combinatorial chemistry 2009, 11 (2), 294-302.

13. Lebl, M.; Krchnák, V.; Sepetov, N. F.; Seligmann, B.; Strop, P.; Felder, S.; Lam, K. S., Onebead-one-structure combinatorial libraries. Biopolymers 1995, 37 (3), 177-98.

14. Gao, Y.; Amar, S.; Pahwa, S.; Fields, G.; Kodadek, T., Rapid Lead Discovery Through Iterative Screening of One Bead One Compound Libraries. ACS Combinatorial Science 2015, 17 (1), 49-59.

15. Paulick, M. G.; Hart, K. M.; Brinner, K. M.; Tjandra, M.; Charych, D. H.; Zuckermann, R. N., Cleavable hydrophilic linker for one-bead-one-compound sequencing of oligomer libraries by tandem mass spectrometry. Journal of combinatorial chemistry 2006, 8 (3), 417-26.

16. Kodadek, T.; McEnaney, P. J., Towards vast libraries of scaffold-diverse, conformationally constrained oligomers. Chemical communications (Cambridge, England) 2016, 52 (36), 6038-59.

17. (a) Youngquist, R. S.; Fuentes, G. R.; Lacey, M. P.; Keough, T., Generation and screening of combinatorial peptide libraries designed for rapid sequencing by mass spectrometry. Journal of the American Chemical Society 1995, 117 (14), 3900-3906; (b) Wang, X.; Peng, L.; Liu, R.; Gill, S. S.; Lam, K. S., Partial Alloc-Deprotection Approach for Ladder Synthesis of "One-Bead OneCompound" Combinatorial Libraries. Journal of combinatorial chemistry 2005, 7 (2), 197-209; (c) Chait, B. T.; Wang, R.; Beavis, R. C.; Kent, S. B., Protein ladder sequencing. Science (New York, N.Y.) 1993, 262 (5130), 89-92; (d) Wang, P.; Arabaci, G.; Pei, D., Rapid Sequencing of LibraryDerived Peptides by Partial Edman Degradation and Mass Spectrometry. Journal of combinatorial chemistry 2001, 3 (3), 251-254.

18. Liu, R.; Marik, J.; Lam, K. S., A Novel Peptide-Based Encoding System for "One-Bead OneCompound" Peptidomimetic and Small Molecule Combinatorial Libraries. Journal of the American Chemical Society 2002, 124 (26), 7678-7680.

19. (a) Liu, X.; Hsieh, H. B.; Campana, D.; Bruce, R. H., A new method for high speed, sensitive detection of minimal residual disease. Cytom Part A 2012, 81A (2), 169-175; (b) Krivacic, R. T.; 
Ladanyi, A.; Curry, D. N.; Hsieh, H. B.; Kuhn, P.; Bergsrud, D. E.; Kepros, J. F.; Barbera, T.; Ho, M. Y.; Chen, L. B.; Lerner, R. A.; Bruce, R. H., A rare-cell detector for cancer. Proceedings of the National Academy of Sciences of the United States of America 2004, 101 (29), 10501-4; (c) Ao, Z.; Liu, X., Fiber-Optic Array Scanning Technology (FAST) for Detection and Molecular Characterization of Circulating Tumor Cells. Methods Mol Biol 2017, 1634, 235-246; (d) Curry, D. N.; Krivacic, R. T.; Hsieh, H. B.; Ladanyi, A.; Bergsrud, D. E.; Ho, M. Y.; Chen, L. B.; Kuhn, P.; Bruce, R. H., High-speed detection of occult tumor cells in peripheral blood. Conf Proc IEEE Eng Med Biol Soc 2004, 2004, 1267-70; (e) DeMaster, L. K.; Liu, X.; VanBelzen, D. J.; Trinité, B.; Zheng, L.; Agosto, L. M.; Migueles, S. A.; Connors, M.; Sambucetti, L.; Levy, D. N.; Pasternak, A. O.; O'Doherty, U., A Subset of CD4/CD8 Double-Negative T Cells Expresses HIV Proteins in Patients on Antiretroviral Therapy. J Virol 2015, 90 (5), 2165-79; (f) Wang, D.; Wu, L.; Liu, X., Glycan Markers as Potential Immunological Targets in Circulating Tumor Cells. Adv Exp Med Biol 2017, 994, 275-284.

20. (a) Alluri, P. G.; Reddy, M. M.; Bachhawat-Sikder, K.; Olivos, H. J.; Kodadek, T., Isolation of protein ligands from large peptoid libraries. J Am Chem Soc 2003, 125 (46), 13995-4004; (b) Olivos, H. J.; Bachhawat-Sikder, K.; Kodadek, T., Quantum dots as a visual aid for screening beadbound combinatorial libraries. Chembiochem : a European journal of chemical biology 2003, 4 (11), 1242-5; (c) Pei, D., On-bead library screening made easier. Chem Biol 2010, 17 (1), 3-4.

21. Liu, X.; Hsieh, H. B.; Campana, D.; Bruce, R. H., A new method for high speed, sensitive detection of minimal residual disease. Cytometry $A$ 2012, 81 (2), 169-75.

22. Chen, X.; Tan, P. H.; Zhang, Y.; Pei, D., On-bead screening of combinatorial libraries: reduction of nonspecific binding by decreasing surface ligand density. Journal of combinatorial chemistry 2009, 11 (4), 604-11.

23. Mitchell, A. R.; Erickson, B. W.; Ryabtsev, M. N.; Hodges, R. S.; Merrifield, R. B., Tertbutoxycarbonylaminoacyl-4-(oxymethyl)-phenylacetamidomethyl-resin, a more acid-resistant support for solid-phase peptide synthesis. J Am Chem Soc 1976, 98 (23), 7357-62.

24. (a) Solid-Phase Synthesis. Boca Raton: CRC Press, 2000; (b) Thieriet, N.; Alsina, J.; Giralt, E.; Guibé, F.; Albericio, F., Use of Alloc-amino acids in solid-phase peptide synthesis. Tandem deprotection-coupling reactions using neutral conditions. Tetrahedron Lett. 1997, 38 (41), 72757278.

25. (a) Zubarev, R. A.; Makarov, A., Orbitrap Mass Spectrometry. Analytical Chemistry 2013, 85 (11), 5288-5296; (b) Hecht, E. S.; Scigelova, M.; Eliuk, S.; Makarov, A., Fundamentals and 
Advances of Orbitrap Mass Spectrometry. In Encyclopedia of Analytical Chemistry, Meyers, R. A., Ed. 2020; pp 1-40.

26. Hsu, F.; Pringle, T. H.; Kuhn, R. M.; Karolchik, D.; Diekhans, M.; Haussler, D.; Kent, W. J., The UCSC Proteome Browser. Nucleic Acids Res 2005, 33 (Database issue), D454-8.

27. Stephen, A. G.; Esposito, D.; Bagni, R. K.; McCormick, F., Dragging ras back in the ring. Cancer Cell 2014, 25 (3), 272-81.

28. Tanowitz, M.; Hettrick, L.; Revenko, A.; Kinberger, G. A.; Prakash, T. P.; Seth, P. P., Asialoglycoprotein receptor 1 mediates productive uptake of $\mathrm{N}$-acetylgalactosamine-conjugated and unconjugated phosphorothioate antisense oligonucleotides into liver hepatocytes. Nucleic Acids Res 2017, 45 (21), 12388-12400.

29. (a) Nair, J. K.; Willoughby, J. L.; Chan, A.; Charisse, K.; Alam, M. R.; Wang, Q.; Hoekstra, M.; Kandasamy, P.; Kel'in, A. V.; Milstein, S.; Taneja, N.; O'Shea, J.; Shaikh, S.; Zhang, L.; van der Sluis, R. J.; Jung, M. E.; Akinc, A.; Hutabarat, R.; Kuchimanchi, S.; Fitzgerald, K.; Zimmermann, T.; van Berkel, T. J.; Maier, M. A.; Rajeev, K. G.; Manoharan, M., Multivalent N-acetylgalactosamineconjugated siRNA localizes in hepatocytes and elicits robust RNAi-mediated gene silencing. J Am Chem Soc 2014, 136 (49), 16958-61; (b) Sanhueza, C. A.; Baksh, M. M.; Thuma, B.; Roy, M. D.; Dutta, S.; Préville, C.; Chrunyk, B. A.; Beaumont, K.; Dullea, R.; Ammirati, M.; Liu, S.; Gebhard, D.; Finley, J. E.; Salatto, C. T.; King-Ahmad, A.; Stock, I.; Atkinson, K.; Reidich, B.; Lin, W.; Kumar, R.; Tu, M.; Menhaji-Klotz, E.; Price, D. A.; Liras, S.; Finn, M. G.; Mascitti, V., Efficient Liver Targeting by Polyvalent Display of a Compact Ligand for the Asialoglycoprotein Receptor. J Am Chem Soc 2017, 139 (9), 3528-3536; (c) Huang, X.; Leroux, J. C.; Castagner, B., Well-Defined Multivalent Ligands for Hepatocytes Targeting via Asialoglycoprotein Receptor. Bioconjug. Chem. 2017, 28 (2), 283-295.

30. Westerlind, U.; Westman, J.; Törnquist, E.; Smith, C. I.; Oscarson, S.; Lahmann, M.; Norberg, T., Ligands of the asialoglycoprotein receptor for targeted gene delivery, part 1: Synthesis of and binding studies with biotinylated cluster glycosides containing $\mathrm{N}$ acetylgalactosamine. Glycoconj. J. 2004, 21 (5), 227-41.

31. Nanjan, P.; Porel, M., Sequence-defined non-natural polymers: synthesis and applications. Polymer Chemistry 2019, 10 (40), 5406-5424. 
32. McGuire, M. J.; Gray, B. P.; Li, S.; Cupka, D.; Byers, L. A.; Wu, L.; Rezaie, S.; Liu, Y. H.; Pattisapu, N.; Issac, J.; Oyama, T.; Diao, L.; Heymach, J. V.; Xie, X. J.; Minna, J. D.; Brown, K. C., Identification and characterization of a suite of tumor targeting peptides for non-small cell lung cancer. Scientific reports 2014, 4, 4480.

33. Liu, F.; Du, D.; Fuller, A. A.; Davoren, J. E.; Wipf, P.; Kelly, J. W.; Gruebele, M., An experimental survey of the transition between two-state and downhill protein folding scenarios. Proceedings of the National Academy of Sciences 2008, 105 (7), 2369-2374.

34. Westerlind, U.; Westman, J.; Törnquist, E.; Smith, C. I. E.; Oscarson, S.; Lahmann, M.; Norberg, T., Ligands of the asialoglycoprotein receptor for targeted gene delivery, part 1: Synthesis of and binding studies with biotinylated cluster glycosides containing $\mathrm{N}$ acetylgalactosamine. Glycoconj. J. 2004, 21 (5), 227-241. 


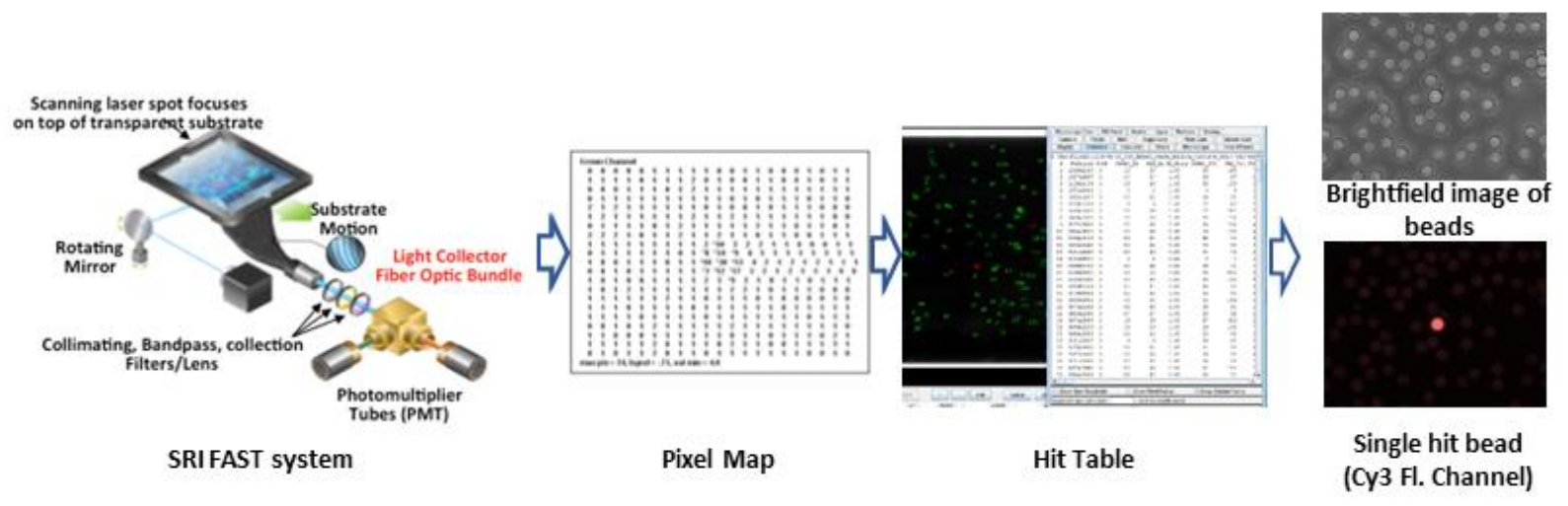

Figure 1. Diagram of the SRI Fiber-optic Array Scanning Technology (FAST) system. The FAST system uses rapid laser scanning with sensitive photomultiplier tube (PMT) fluorescence emission detection to rapidly generate a pixel map indicating the position of fluorescently labeled beads. Analysis of the pixel map generates a hit table with cartesian coordinates and multiple calculated fluorescence metrics to detect hit beads with high sensitivity and specificity. The coordinates of the hits can then be transferred to other microscopy systems for additional multi-wavelength imaging analysis or bead extraction. 
a.

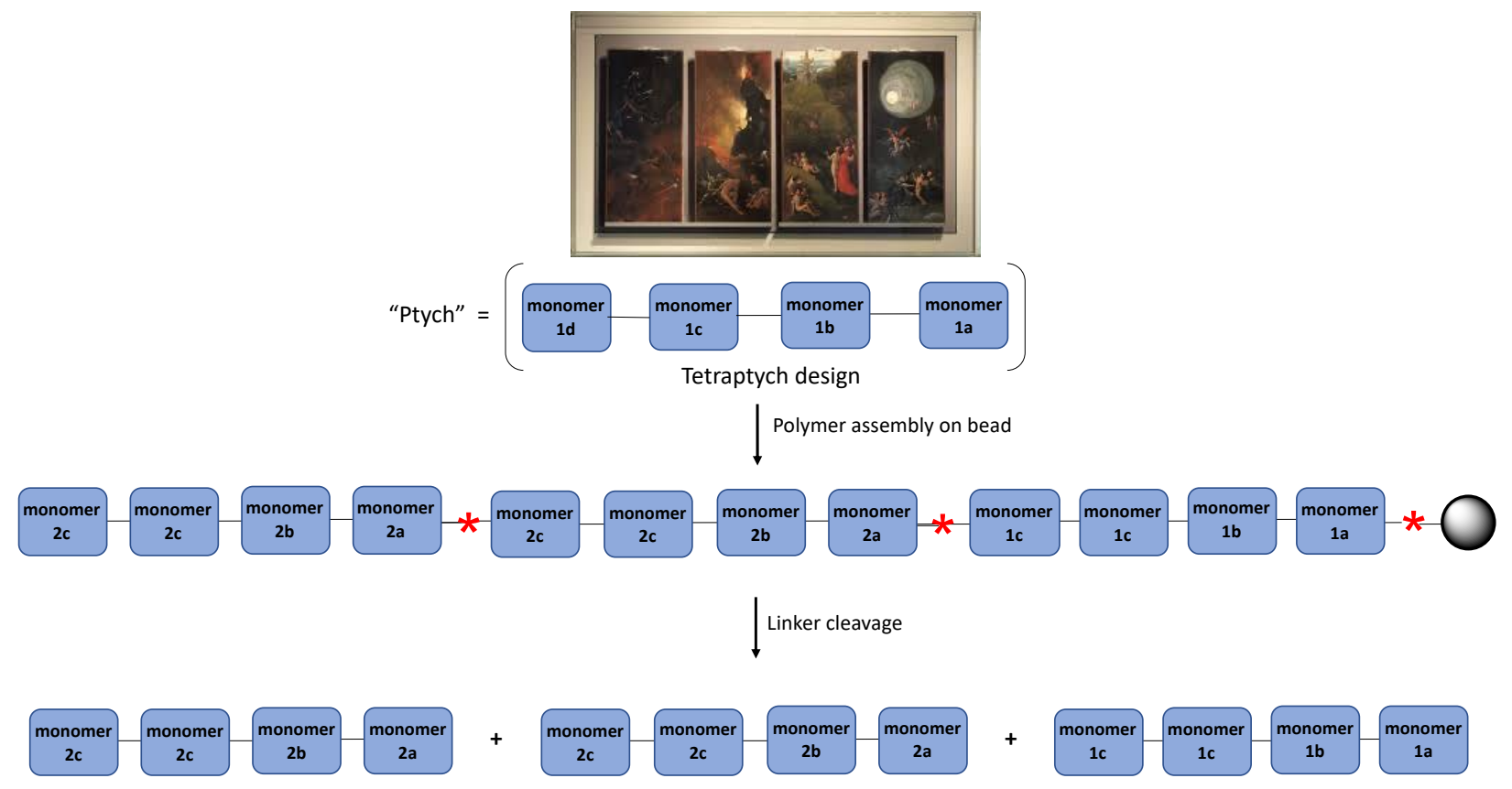

b.

$9 \mathbf{c}-9 \mathbf{b}-9 \mathbf{a} \pi_{0}^{0} \ldots \ldots, \ldots$ $\downarrow \mathrm{NH}_{4} \mathrm{OH}_{\text {(aq) }}$

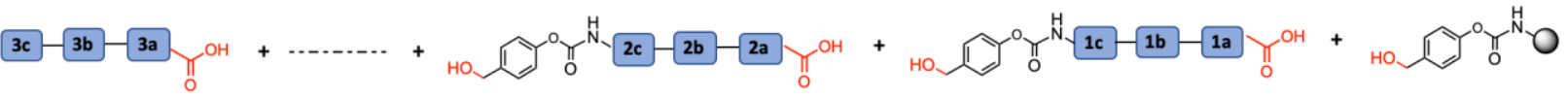

Figure 2. Description of ptych design. (a) Example of a tetraptych painting (visioni dell'aldilà [Visions of the Hereafter] by Hieronymus Bosch) and the analogous ptych design of the polymers. Each polymer is constructed of a sequence of multiple tetrapytchs each consisting of four diverse monomers and a cleavable linker $(*)$. During sequencing each polymer is subjected to a chemical cleavage reaction in 
which the linkers are cleaved (at the $*$ position) to generate a mixture of the ptych fragments. (b) Tetraptych-based polymer with PAM (phenyl-acetamido-methylene) as the cleavable linker (shown in black), and the cleavable ester linker shown in red. Once subjected to a solution of ammonium hydroxide, the ester linkers are cleaved to generate a mixture of all the tetraptychs constituting the polymer which can be each identified to reconstruct the full polymer sequence. 
a.

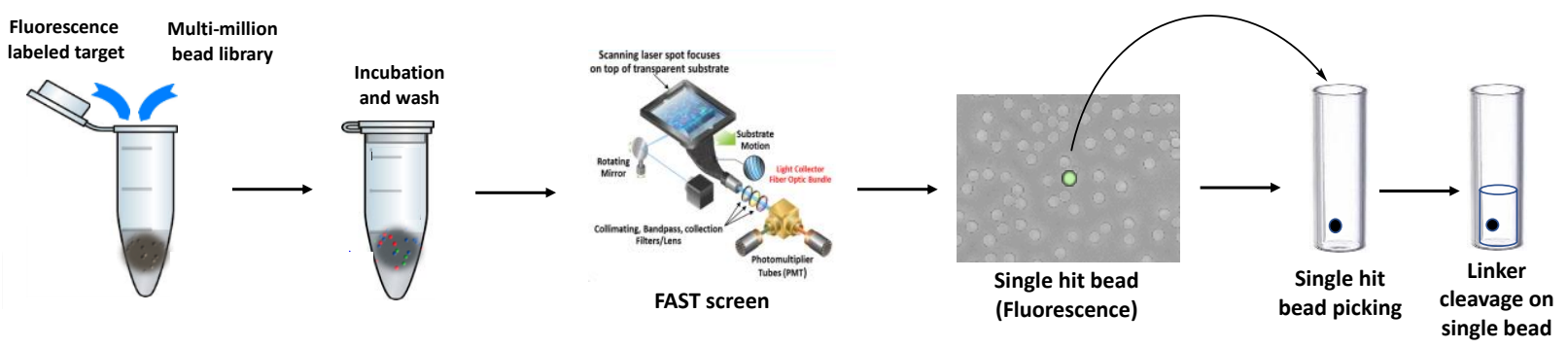

b.

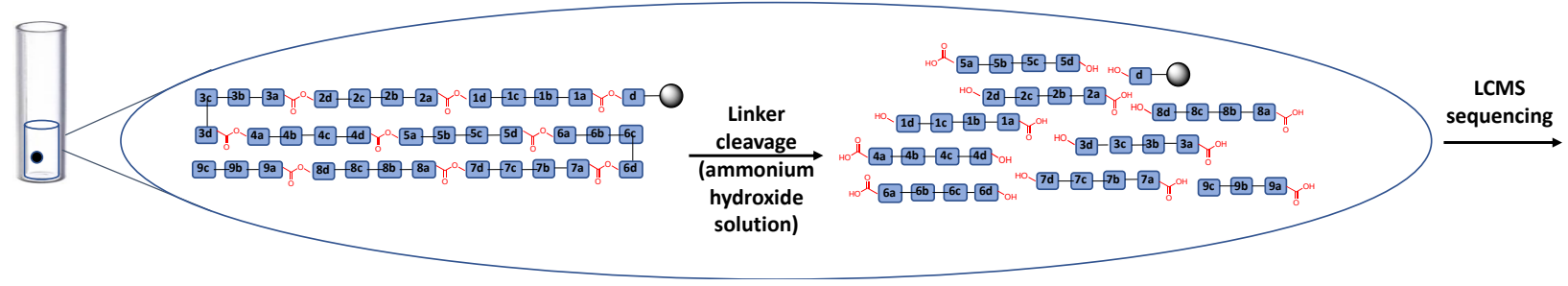

c.

Ptych Position

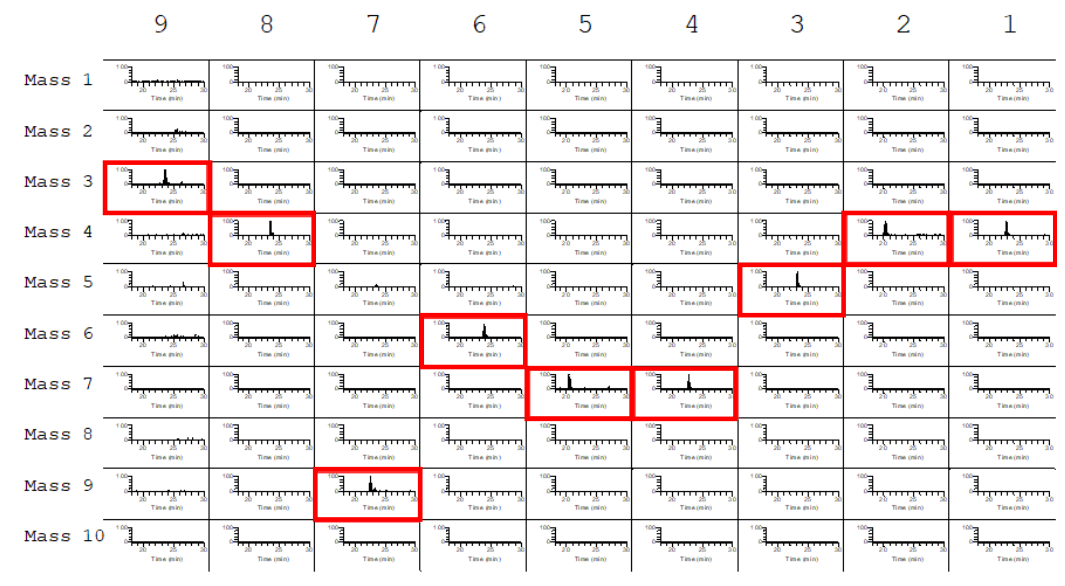


d.

Fluorescein-(D)Asn-(D)Val-(L)Phe-PAM-(D)Tyr-(D)Ser-(L)Val-PAM-(D)Arg-(D)Ser-(L)Phe-PAM(D)Phe-(D)Arg-(L)Ala-PAM-(D)Tyr-(D)Lys-(L)Ala-PAM-(D)Glu-(D)Arg-(L)Leu-PAM-(D)Ala-(D)Arg(L)Leu-PAM-(D)Pro-(D)Arg-(L)Ala-PAM-(D)Phe-(D)Lys-Gly-PAM

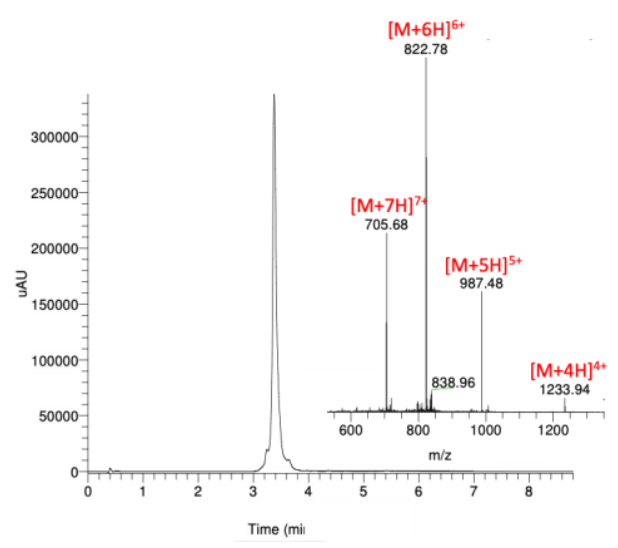

Figure 3. Summary of the screening process of a bead-based library. (a) The polymer-bead library is incubated with a fluorescently labeled target of interest, washed and then screened by FAST to identify the location of positive hits. Hit beads are evaluated by bright field and fluorescence microscopy and confirmed bead hits are picked into a separate LC-MS vials and subjected to the cleavage solution. (b) Upon treatment with ammonium hydroxide, all the esters are hydrolyzed to yield a mixture of the different tetraptychs of a single sequence in each vial. (c) Analysis of ptych fragment masses allows reconstruction of the ptychs into a specific sequence based on the library design. (d) LC-MS chromatogram of a purified full-length 36-mer (9 tetraptych) polymer of which the sequence analysis is shown in Figure 4c. The compound hit was characterized by LC-MS (ESI); observed: $4931.9 \pm 0.9$ Da; calculated: 4932.5 Da. 
a.

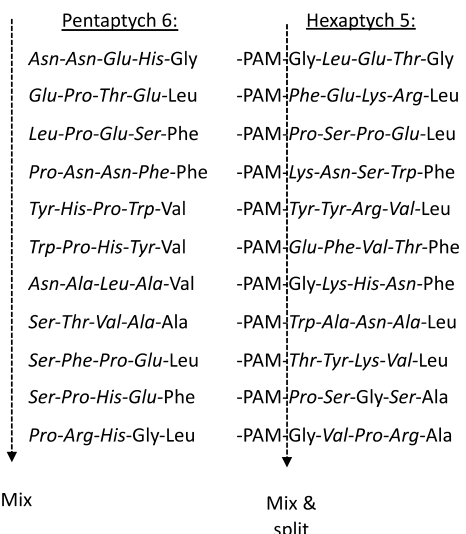

split
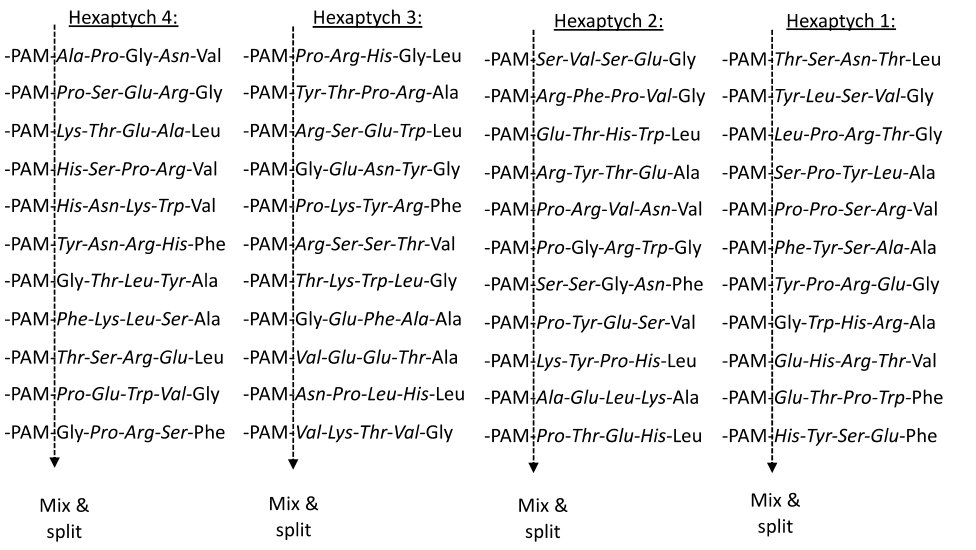

PAM

-PAM

-PAM

-PAM

-PAM

-PAM - $\mathrm{O}$

-PAM

-PAM

-PAM

-PAM -PAM

b.
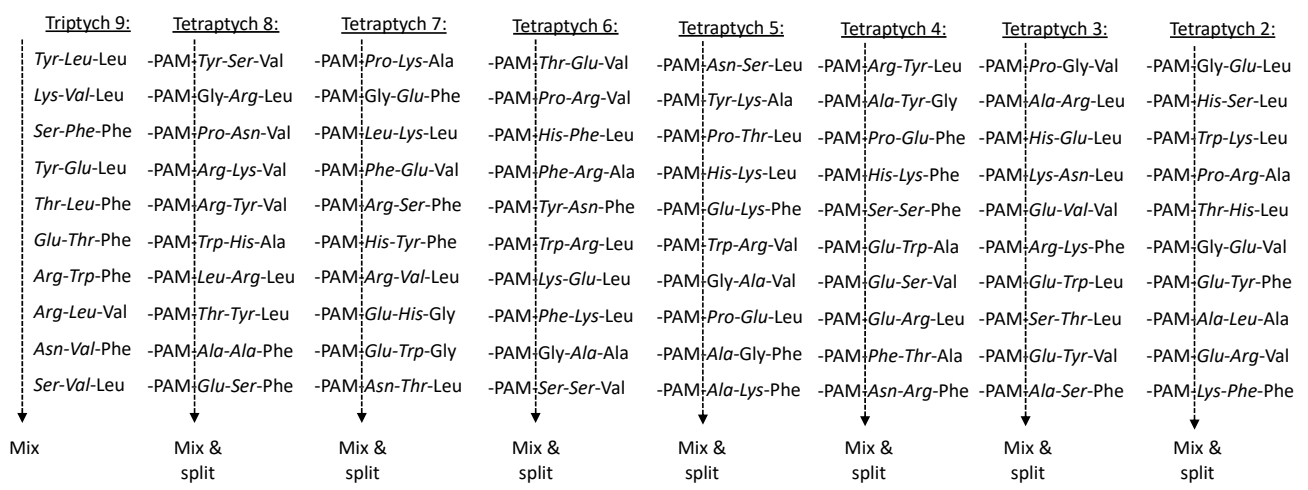

Tetraptych 1: -PAM Ala-Val-Leu -PAM -PAM His-Tyr-Val -PAM -PAMThr-Ser-Val -PAM -PAM Phe-Lys-Gly -PAM -PAM Thr-Glu-Leu -PAM -PAM-Phe-Arg-Gly -PAM -PAM-Trp-Glu-Val -PAM -PAMTyr-Arg-Phe -PAM -PAM Pro-Ala-Ala -PAM -PAM His-Glu-Val -PAM Mix \& split split

split

split

Figure 4. Libraries design and validation. (a) Hexaptych design for the $\sim 1.77$ million compound library NNP1. (b) Tetraptych design for the 1 billion compound library NNP2. $O_{\text {represents resin beads. Italics }}$ indicates D-amino acid. In our design there are D-amino acids and PAM linker, which are both non-natural building blocks. We chose to use L-amino acids at the position next to PAM as the corresponding fmoc-Lamino acid-PAMs were commercially available, whereas the D form was not. 
a.

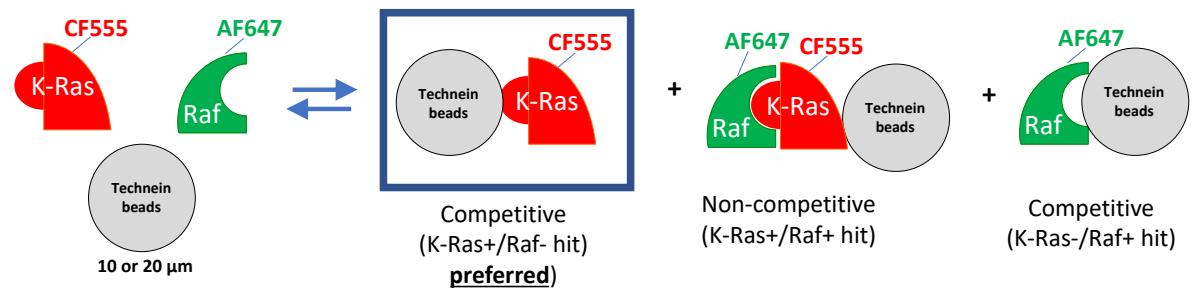

b.

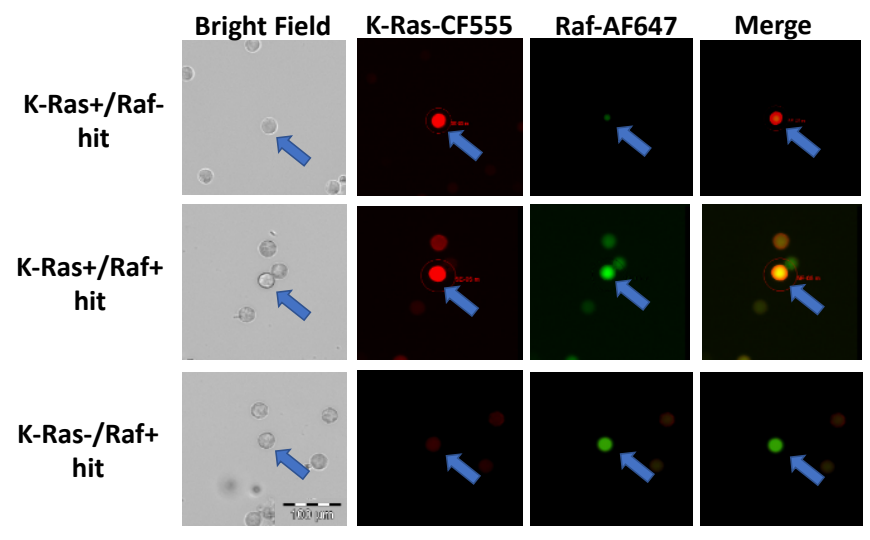

c.
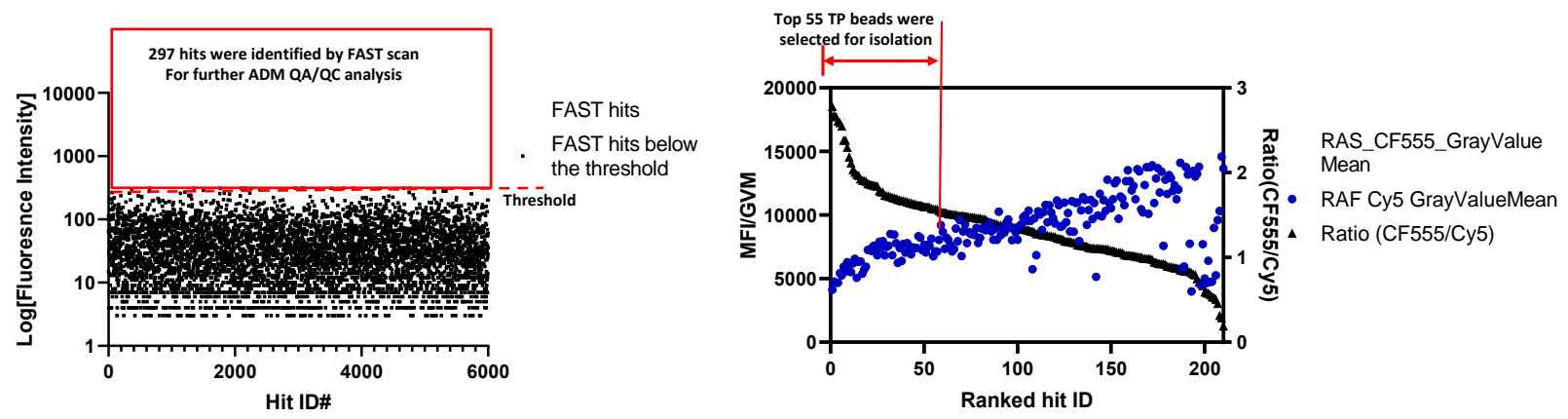
d.
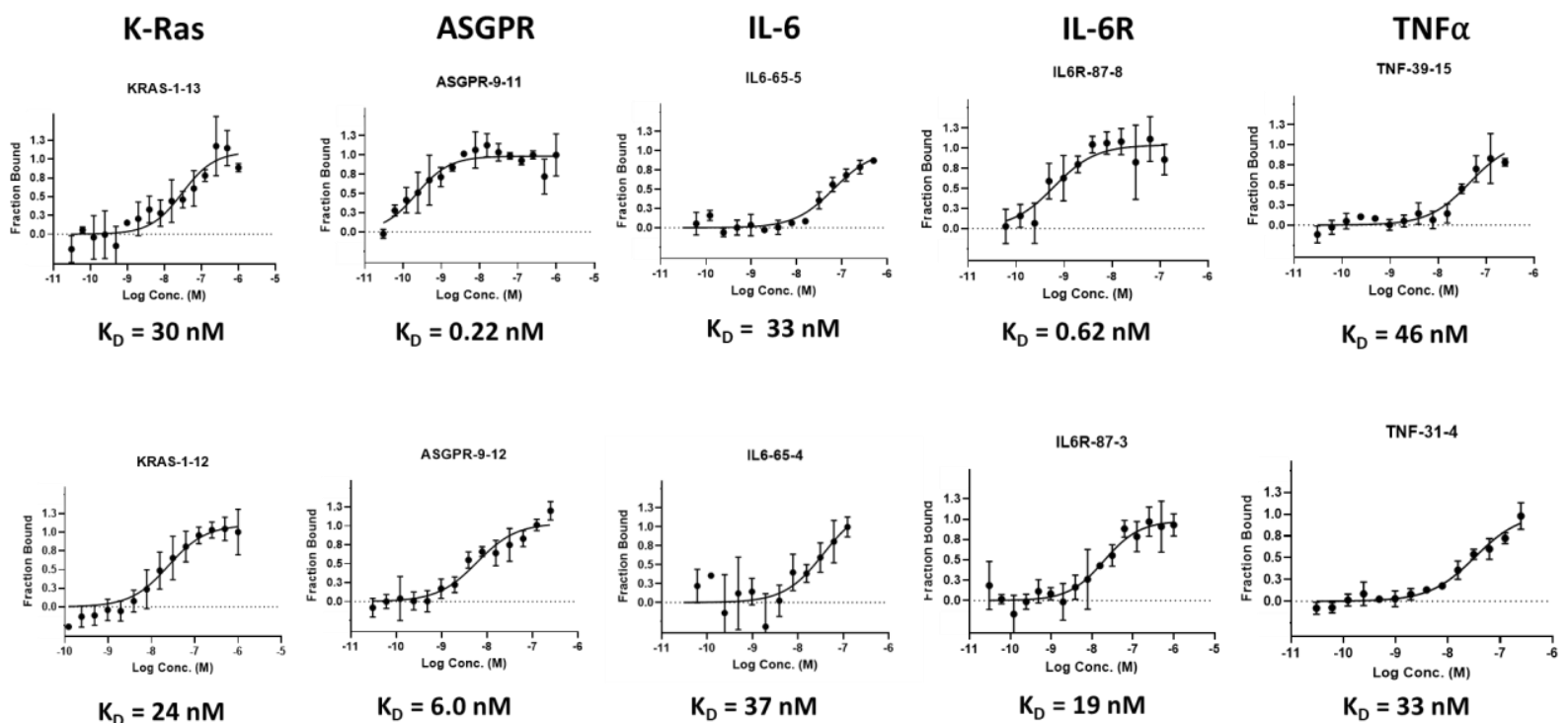

Figure 5. Screening data and representative MST data. (a) Competition screen of library beads against the primary target K-Ras labeled with CF555 in the presence of Raf-RBD as a counter target labeled with Alexa Fluor 647 (AF647). Only the single positive beads with fluorescence at $555 \mathrm{~nm}$ were picked and sequenced. This strategy was performed to enrich for inhibitors that would bind to K-Ras while blocking its interaction with its downstream signaling partner Raf. (b) Automated digital microscopic images demonstrating three types of hits. Top: single positive bead binds the primary target K-Ras but not the counter target Raf. Middle: double single positive bead binds both the primary target K-Ras and the counter target Raf. Bottom: single positive bead binds the counter target Raf but not the primary target K-Ras. (c) Hit deification by rapid FAST screen for K-Ras binding NNPs. Left: After FAST scan, the software filters out false positive hits including autofluorescence particles using the dual-wavelength comparison technology. 300 top hits with bright KRas- CF555 (in red) that above the threshold were identified for further ADM/CellCelector imaging and analysis from a 2.5 million 20-um bead sample plate. Right: The FAST hits identified in A were imaged, reviewed and analyzed on Cellcelector at high resolution. The MFI were measured for each true positive (TP) bead and the top ranked 55 TP hits based on high MFI of K-Ras- 
CF555 and ratio (CF555/Cy5) were isolated for sequencing, resynthesis and characterization. (d) Representative of MST data showing the binding of resynthesized purified NNPs to their targets and the calculated $K_{D}$ values. 
a.

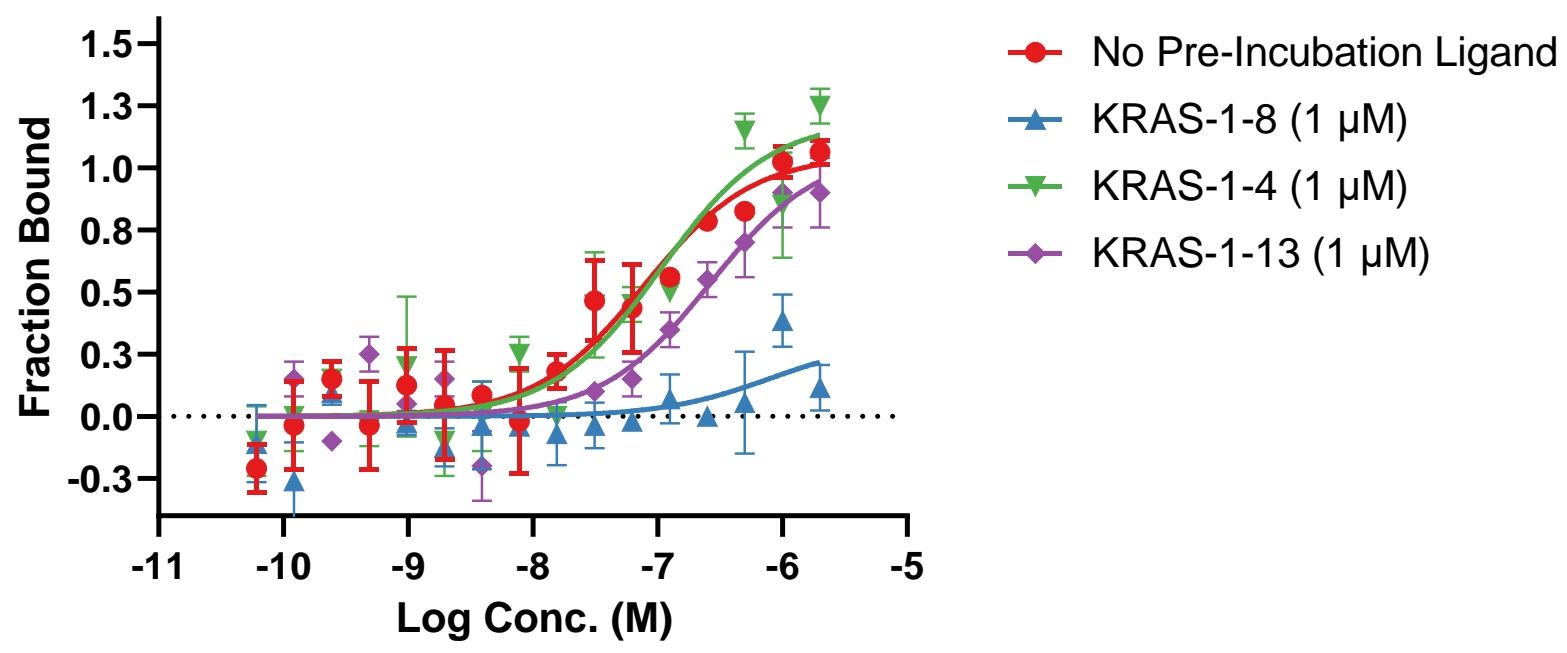

b.
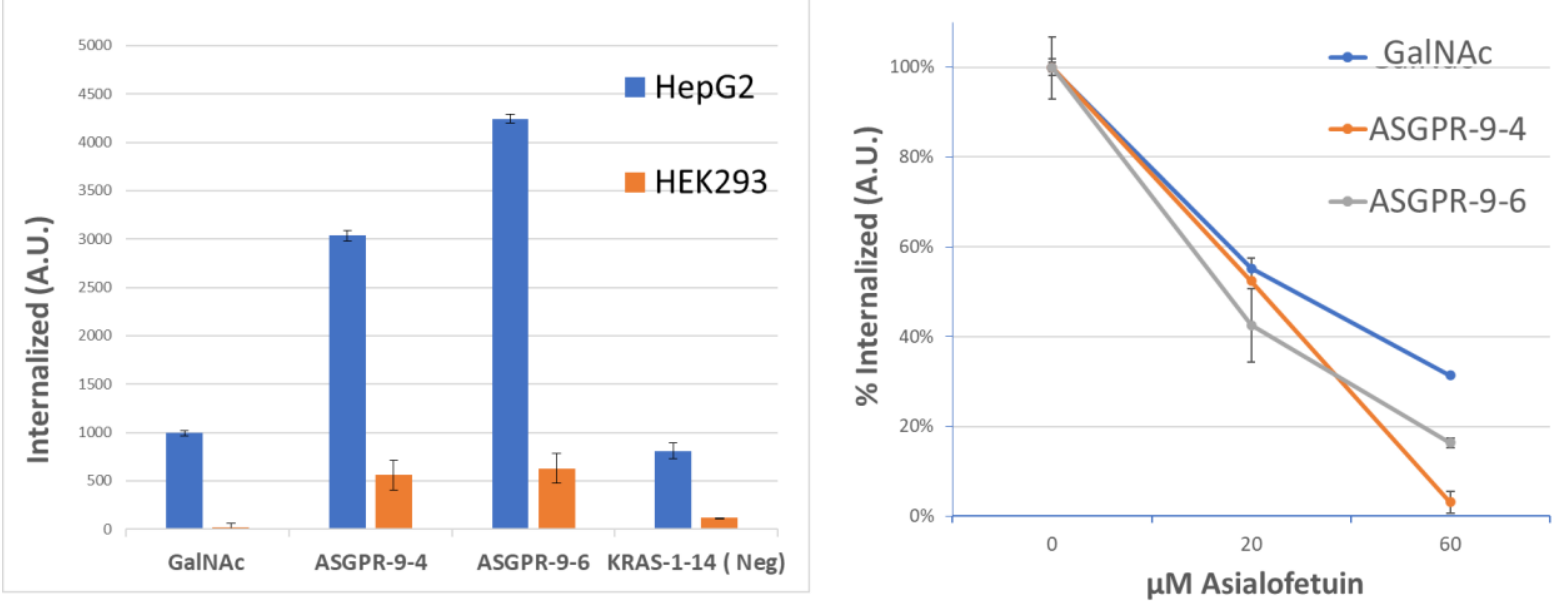

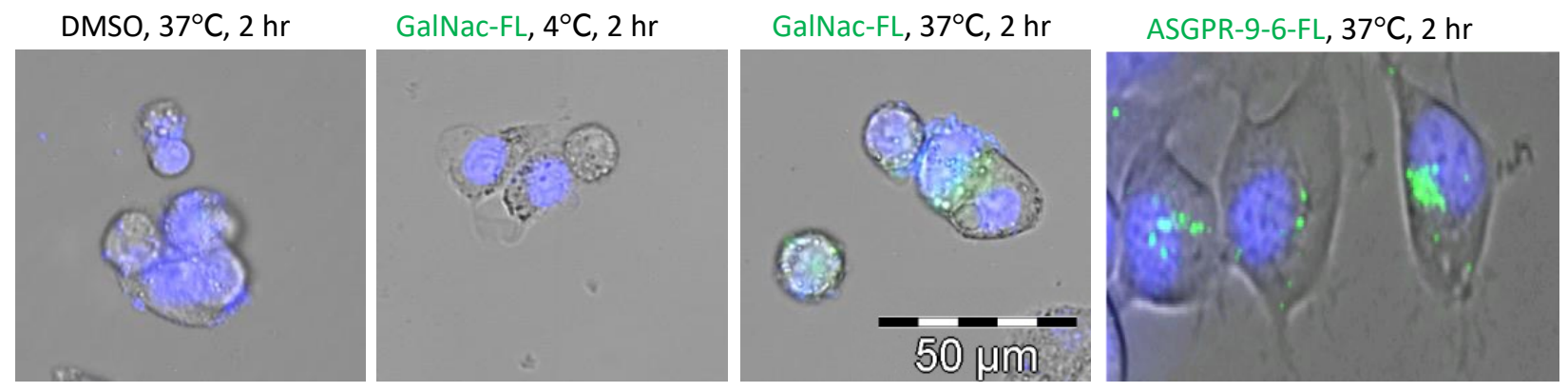

c.
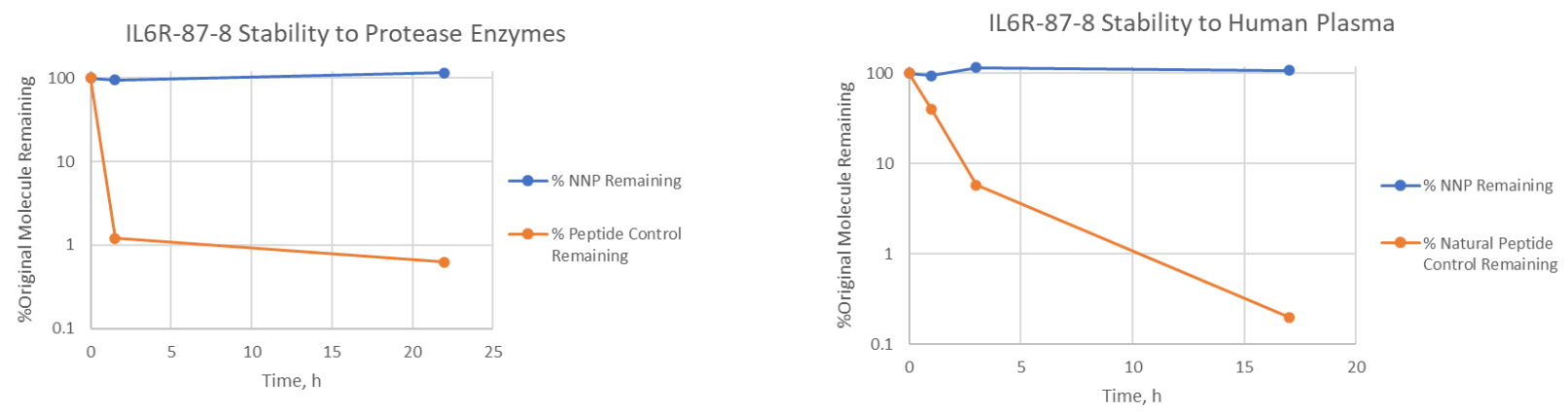

Figure 6. Biological relevance and stability of NNPs in biological matrices. (a) K-Ras - Raf interaction was measured by MST using a fixed concentration of K-Ras $(5 \mathrm{nM})$ and a titration of Raf to give a $78 \mathrm{nM}$ binding affinity. Competitive inhibition of the K-Ras - Raf protein-protein interaction was tested with a 15 minute pre-incubation with NNP ligands ( $1 \mu \mathrm{M}$ concentration) followed by titration with Raf to measure binding by MST. NNP KRAS-1-8 showed complete inhibition, NNP KRAS-1-13 caused a shift in the $K_{D}\left(K_{D}=260 \mathrm{nM}\right)$, and NNP KRAS-1-4 showed no inhibition $\left(K_{D}=100 \mathrm{nM}\right)$. (b) Left: cell uptake of GalNAc (positive control), two NNP hits (ASGPR-9-4 and ASGPR-9-6) and a non-hit NNP (KRAS-1-14, negative control) in HepG2 (high ASGPR expressing) vs. HEK293 (low ASGPR expressing) cells lines. Right: competition uptake assay of GalNAc and two NNP hits (ASGPR-9-4 and ASGPR-9-6) in HepG2 cells after preincubation with different concentrations of asialofetuin (a naturally occurring serum protein ASGPR ligand). Decrease in cell uptake with increasing concentrations of asialofetuin indicates blocking of ASGPR-mediated uptake. Lower: Confocal images of HepG2 cell uptake (scale: x20 for the three left images and x40 for the right image). FL 
= fluorescein label; Cell nucleus staining with DAPI (blue). No uptake with the blank sample (DMSO) is seen at $4^{\circ} \mathrm{C}$. Also, no uptake of the control GaINAc is seen at $4{ }^{\circ} \mathrm{C}$ but is seen at $37{ }^{\circ} \mathrm{C}$. Similarly intracellular uptake is observed for the NNP hit (ASGPR-9-6) at $37{ }^{\circ} \mathrm{C}$ (but not at $4{ }^{\circ} \mathrm{C}$ - not shown). (c) Stability data for NNP IL6R-87-8 compared to its $L$ variant in the presence of proteinase $K$ (left) and for the same NNP in human plasma (right) compared with Angiotensin I. 